\title{
Probabilistic Relevance Relations
}

\author{
Dan Geiger and David Heckerman
}

\begin{abstract}
The intuition behind the construction of Bayesian networks and other graph-based representations of joint probability distributions from expert judgments is based on the assumed relationship between "connectedness" in the graphical model and "relatedness" among the variables involved. We show that several plausible definitions of relatedness do not adhere to such an equivalence. We then provide a definition of probabilistic relatedness that is closely related to connectedness in the graphical model and prove that the two concepts are equivalent whenever the model uses only propositional variables and assuming every combination of value assignment to these variables is feasible. We conjecture that the equivalence established holds also when these restrictions are lifted.
\end{abstract}

Index Terms-Bayesian networks, conditional independence.

\section{INTRODUCTION}

B AYESIAN networks are graph-based representations of joint probability distributions which have found a variety of applications for diagnosis, prediction, image recovery, and in many other domains [9]. There are three options for constructing Bayesian networks. The first is to build a network manually with the help of a domain expert. This approach is used quite often and is most useful for moderate-size models. A second approach is to construct a Bayesian network completely from data. This approach is most useful when a database of cases is available and when experts are too costly or unavailable. Finally, a hybrid method by which a rough model is build from expert's judgments and then tuned by data is perhaps the most promising approach. The analysis of this paper concentrates on issues arising from the construction of Bayesian networks from expert's judgments.

To be concrete, let us first consider a simple Bayesian network $D_{0}$ of the form $a \rightarrow c \leftarrow b$. This network represents a joint probability distribution $p(a, b, c)$ of three random variables $a, b$, and $c$, such that $p(a, b, c)=p(a) p(b) p(c \mid a, b)$. It is a minimal Bayesian network of $p$ if none of it edges can be removed, that is, neither $p(a, b, c)=p(a) p(b) p(c \mid a)$ nor $p(a, b, c)=p(a) p(b) p(c \mid b)$ hold for all assignments for $a, b$, and $c$.

The intuition behind the construction of Bayesian networks from expert judgments is based on the assumed relationship between "connectedness" in the graphical model and "relatedness" between the variables involved. That is, for example, $a$

Manuscript received February 19, 1995; revised August 11, 1997. An earlier version of this paper was presented at [4].

D. Geiger is with Microsoft Research, Redmond, WA 98052-6399 USA, on leave from the Department of Computer Science, Technion-Israel Institute of Technology, Haifa, Israel, 32000 (e-mail: dang@cs.technion.ac.il).

D. Heckerman is with Microsoft Research, Redmond, WA 98052-6399 USA (e-mail: heckerma@microsoft.com).

Publisher Item Identifier S 1083-4427(98)00124-6. and $b$ are connected in $D_{0}$ because they are related to each other through $c$. However, one can easily construct examples of a distribution $p$ such that $D_{0}$ is a minimal network of $p$ yet $a$ and $b$ are marginally independent, and also conditionally independent given any specific value of $c$. Such an example seemingly contradicts the analogy between "connectedness" in the graphical model and "relatedness" in the joint distribution because $a$ and $b$ are seemingly unrelated in any context - when $c$ is unknown and when $c$ is known-yet they are connected in the graphical representation.

In this paper, we seek a definition of relatedness that fits the intuition that connected nodes in the graphical representation correspond to variables that are related probabilistically. We shall prove that our concept of relatedness is indeed equivalent to connectedness in a minimal Bayesian network under the assumption that all variables are propositional and that every combination of value assignment is feasible. We conjecture that the analogy established holds even when these restrictions are lifted and hope that this paper will stimulate the resolution of this conjecture

Apart from the epistemological reassurance given by our definition, our results also justify prevailing decomposition techniques that simplify the process of acquiring probabilistic knowledge from domain experts via models known as similarity networks [7]. A similarity network is a set of Bayesian networks, called the local networks, each constructed under a different set of hypotheses $H_{i}$. In each local network $D_{i}$, only those variables that "help to distinguish" between the hypotheses in $H_{i}$ are depicted. The success of this model stems from the fact that only a small portion of variables helps to distinguish between the carefully chosen set of hypotheses $H_{i}$. Thus, the model usually includes several small networks instead of a single large Bayesian network. A plausible formal definition of what is meant by "help to distinguish" is provided herein, where $x$ helps to distinguish values of $h$ means that $a$ and $h$ are related probabilistically.

This paper is organized as follows. In Section II, we provide definitions of a Bayesian network and conditional independence and review some of their properties. In Section III, we associate connectedness with conditional independence. In Section IV, we develop a definition of probabilistic relatedness, and in Section V we prove the equivalence between relatedness and connectedness under some restrictions.

\section{BACKGROUND}

Throughout the discussion we consider a finite set of variables $U=\left\{u_{1}, \cdots, u_{n}\right\}$ each with a finite domain $d\left(u_{i}\right)$ and a probability distribution $p(U)$ having the Cartesian 
product $\times_{u_{i} \in U} d\left(u_{i}\right)$ as its sample space. We use lowercase letters possibly subscripted (e.g., $a, b, x$, or $u_{i}$ ) to denote variables, and use uppercase letters (e.g., $X, Y$, or $Z$ ) to denote sets of variables. A bold lowercase or uppercase letter refers to a value (instance) of a variable or of a set of variables, respectively. A value $\mathbf{X}$ of a set of variables $X$ is an element in the Cartesian product $\times_{x \in X} d(x)$, where $d(x)$ is the set of values of $x$. The notation $X=\mathrm{X}$ stands for $x_{1}=\mathrm{x}_{1}, \cdots, x_{n}=\mathrm{x}_{n}$, where $X=\left\{x_{1}, \cdots, x_{n}\right\}$ and $\mathrm{x}_{i}$ is a value of $x_{i}$.

We let $X \perp Y \mid Z=\mathrm{Z}$ denote the statement that $X$ and $Y$ are conditionally independent given $Z=\mathbf{Z}$, namely, that $p(X, Y, Z=\mathbf{Z}) p(Z=\mathbf{Z})=p(X, Z=\mathbf{Z}) p(Y, Z=\mathbf{Z})$ for every value of $X$ and $Y$. We let $X \perp Y \mid Z$ denote the statement that $X$ and $Y$ are independent given every value for $Z$, namely, that $X \perp Y \mid Z=\mathbf{Z}$ holds for every $\mathbf{Z}$. Similarly, $X \perp Y$ denotes the statement $X$ and $Y$ are marginally independent which can also be thought of as a shorthand notation for $X \perp Y \mid \emptyset$.

A Bayesian network is a representation of independence statements as well as a representation of a joint probability distribution. Below we give a definition and some consequences. For a more comprehensive overview, consult [9].

Definition [9]: A directed acyclic graph $D$ of a joint probability distribution $p(U)$ is a Bayesian network of $p$ if $D$ is constructed from $p$ by the following steps: assign an arbitrary construction order $u_{1}, u_{2},, \cdots, u_{n}$ to the variables in $U$, and designate a node $u_{i}$ for each variable $u_{i} \cdot{ }^{1}$ For each $u_{i}$ in $U$, identify a set $C_{i} \subseteq\left\{u_{1}, \cdots, u_{i-1}\right\}$ such that

$$
\left\{u_{i}\right\} \perp\left\{u_{1}, \cdots, u_{i-1}\right\} \backslash C_{i} \mid C_{i}
$$

holds wrt $p$ (with respect to $p$ ). Assign a link from every node in $C_{i}$ to $u_{i}$. Each node is associated with the conditional probability distribution $p\left(u_{i} \mid C_{i}\right)$. The resulting network is minimal if, for each $u_{i} \in U$, no proper subset of $C_{i}$ satisfies (1).

By the chaining rule it follows that

$$
p\left(u_{1}, \cdots, u_{n}\right)=\prod p\left(u_{i} \mid u_{1}, \cdots, u_{i-1}\right)
$$

and by the definition of $\left\{u_{i}\right\} \perp\left\{u_{1}, \cdots, u_{i-1}\right\} \backslash C_{i} \mid C_{i}$ we further obtain

$$
p\left(u_{1}, \cdots, u_{n}\right)=\prod p\left(u_{i} \mid C_{i}\right) .
$$

Thus, the joint distribution is represented by the network and can be used for computing the posterior probability of every variable given a value to some other variables.

Note that the number of parameters that a Bayesian network requires and the complexity of its topology depend on the construction order, which is not dictated by its definition. There are many possibilities to choose a construction order. In practice, cause-and-effect and time-order relationships often suggest construction orders that yield simple networks. ${ }^{2}$

\footnotetext{
${ }^{1}$ We deliberately denote with $u_{i}$ the node that corresponds to variable $u_{i}$. It will be clear from the context whether we talk about a node or a variable.

${ }^{2}$ Bayesian networks are often called causal networks.
}

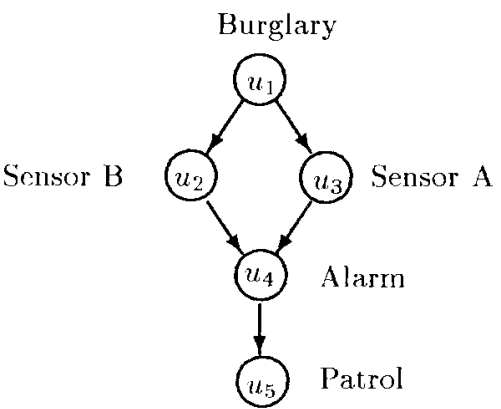

Fig. 1. An example of a Bayesian network

For example, a Bayesian network can represent the following situation. Suppose an alarm system is installed in your house in order to detect burglaries; and suppose it can be activated by two separate sensors. Suppose also that, when the alarm sound is activated, there is a good chance that a police patrol will show up. We are interested in computing the probability of a burglary given a police car is near your house.

We consider five binary variables, burglary $\left(u_{1}\right)$, sensorA $\left(u_{2}\right)$, sensorB $\left(u_{3}\right)$, alarm $\left(u_{4}\right)$, and patrol $\left(u_{5}\right)$, each having two values yes and no. We know that the outcome of the two sensors are conditionally independent given burglary, and that alarm is conditionally independent of burglary given the outcome of the sensors. We also know that patrol is conditionally independent of burglary given alarm (assuming that only the alarm prompts a police patrol). This qualitative information implies that the following three independence statements hold in any probability distribution that describes this story: $\left\{u_{3}\right\} \perp\left\{u_{2}\right\}\left|\left\{u_{1}\right\}\left\{u_{4}\right\} \perp\left\{u_{1}\right\}\right|\left\{u_{2}, u_{3}\right\}$ $\left\{u_{5}\right\} \perp\left\{u_{1}, u_{2}, u_{3}\right\} \mid\left\{u_{4}\right\}$. Consequently, according to our definition, the graph shown in Fig. 1 is a Bayesian network of the burglary story.

In addition to the topology of the network, we need to specify the following conditional distributions: $p\left(u_{1}\right), p\left(u_{2} \mid u_{1}\right)$, $p\left(u_{3} \mid u_{1}\right), p\left(u_{4} \mid u_{2}, u_{3}\right)$, and $p\left(u_{5} \mid u_{4}\right)$. From these conditional distributions, we can compute via (2) any probability involving these variables. However, to do such computations efficiently we need to know additional independence statements which follow from the topology of the network but were not used to construct the Bayesian network (such as $\{$ patrol $\} \perp\{$ burglary $\} \mid\{$ sensorA, sensorB $\}$ ). The criteria of $d$-separation, defined below, provides the most general mechanism to infer independence statements from the topology of the Bayesian network. Some terminology is first established.

A trail in a Bayesian network $D$ is a path in $D$ in which links are taken regardless of their direction. A node $b$ is called a head-to-head node wrt a trail $t$ if there are two consecutive links $a \rightarrow b$ and $b \leftarrow c$ on $t$. Two nodes are connected in a Bayesian network if there exists a trail connecting them. Otherwise they are disconnected. A connected component $C$ of a Bayesian network $D$ is a subgraph of $D$ in which every two nodes are connected. A connected component is maximal if there exists no proper super-graph of $C$ that is a connected component of $D$. If $x \rightarrow y$ is a link in a Bayesian network, 
then $x$ is a parent of $y$ and $y$ is a child of $x$. If there is a directed path from $x$ to $y$, then $x$ is an ancestor of $y$ and $y$ is a descendant of $x$.

Definition [9]: A trail $t$ is active wrt a set of nodes $Z$ if 1) every head-to-head node wrt $t$ either is in $Z$ or has a descendant in $Z$ and 2) every other node along $t$ is outside $Z$. Otherwise, the trail is said to be blocked (or $d$-separated) by $Z$.

In Fig. 1, for example, both trails between $\left\{u_{2}\right\}$ and $\left\{u_{3}\right\}$ are $d$-separated by $Z=\left\{u_{1}\right\}$; the trail $u_{2} \leftarrow u_{1} \rightarrow u_{3}$ is $d$-separated by $Z$ because node $u_{1}$, which is not a head-tohead node wrt this trail, is in $Z$. The trail $u_{2} \rightarrow u_{4} \leftarrow u_{3}$ is $d$-separated by $Z$, because node $u_{4}$ and its descendant $u_{5}$ are outside $Z$. In contrast, $u_{2} \rightarrow u_{4} \leftarrow u_{3}$ is not $d$-separated by $Z^{\prime}=\left\{u_{1}, u_{5}\right\}$ because $u_{5}$ is in $Z^{\prime}$.

The theorem below is the major building block for most of the developments presented in this article and is fundamental to the theory of Bayesian networks.

Theorem 1 [13]: Let $D$ be a Bayesian network of a probability distribution $p(U)$ and let $X, Y$, and $Z$ be three disjoint subsets of $U$. If all trails between a node in $X$ and a node in $Y$ are $d$-separated by $Z$, then $X \perp Y \mid Z$ holds wrt $p$.

For example, in the Bayesian network of Fig. 1, all trails between $u_{1}$ and $u_{\tilde{5}}$ are $d$-separated by $\left\{u_{2}, u_{4}\right\}$. Thus, Theorem 1 guarantees that $\left\{u_{5}\right\} \perp\left\{u_{1}\right\} \mid\left\{u_{2}, u_{3}\right\}$ holds wrt $p$. Geiger et al. [5] generalize Theorem 1 and show that no other graphical criteria reveals more independence statements of $p$ than does $d$-separation. Lauritzen et al. [8] establish another graphical criteria and show that it is equivalent to $d$-separation.

One immediate consequence of Theorem 1 is that if two sets of nodes $X$ and $Y$ are disconnected in a Bayesian network of $p\left(u_{1}, \cdots, u_{n}\right)$, then $X \perp Y$ holds (wrt $p$ ) because there is no active trail between a node in $X$ and a node in $Y$. Another well-known consequence is that if $Z_{a}$ is the set of parents of a node $a$, and $Y_{a}$ are the set of all nodes that are not descendants of $a$ except $a$ 's parents. Then, $\{a\} \perp Y_{a} \mid Z_{a}$ holds (wrt $p$ ). The argument is simple. The set $Z_{a} d$-separates all trails between a node in $Y_{a}$ and $a$ because each such trail either passes through a parent of $a$ and therefore is blocked by $Z_{a}$, or each such trail must reach $a$ through one of $a$ 's children and thus must contain a head-to-head node $w$, where neither $w$ nor its descendants are in $Z_{a}$.

In our proofs we will only use the following properties of conditional independence. A variant of these properties was introduced by Dawid [1] and Spohn [12] and further studied by Pearl [9] and Pearl and Paz [10].

Symmetry

$$
X \perp Y|Z \Rightarrow Y \perp X| Z
$$

Decomposition

$$
X \perp(Y \cup W)|Z \Rightarrow X \perp Y| Z
$$

Weak union

$$
X \perp(Y \cup W)|Z \Rightarrow X \perp Y|(Z \cup W)
$$

Contraction

$$
X \perp Y|Z \& X \perp W|(Z \cup Y) \Rightarrow X \perp(Y \cup W \mid Z) .
$$

It is worth mentioning that the proof of Theorem 1 only uses these properties and therefore every trinary relation $\perp$ that satisfies these properties can be represented by a Bayesian network and the result of Theorem 1 applies. In particular, partial correlation and embedded multivalued dependencies (from relational database theory) satisfy these properties.

\section{CONNECTEDNESS IN TERMS OF INDEPENDENCE}

In this section, we show that if $a$ and $b$ are disconnected in one minimal network of $p$ then $a$ and $b$ are disconnected in every minimal network of $p$. This result shows that the concept of connectivity can be phrased in terms of independence statements that hold in $p$. Indeed, we find that $a$ and $b$ are disconnected in a minimal Bayesian network of $p(U)$ if and only if there exists a partition $U_{a}, U_{b}$ of $U$ such that $U_{a} \perp U_{b}$, $a \in U_{a}$ and $b \in U_{b}$ (a partition of a set $U$ is a pair of nonempty disjoint subsets of $U$ whose union is $U$ ).

Lemma 2: Let $D$ be a minimal Bayesian network of $p(U)$, and $D_{X}$ be a connected component of $D$ with a set of nodes $X$. Then, there exists no partition $X_{1}, X_{2}$ of $X$ such that $X_{1} \perp X_{2}$ holds wrt $p$.

Proof: Assume contrary to the lemma's claim that $X_{1}, X_{2}$ is a partition of $X$ and that $X_{1} \perp X_{2}$ holds. Since $X_{1}$ and $X_{2}$ are connected in $D$, there must exist a link between a node in $X_{1}$ and a node in $X_{2}$. Without loss of generality, assume it is directed from a node $v$ in $X_{1}$ to a node $u$ in $X_{2}$. Let $Z_{1}, Z_{2}$ be the parents of $u$ in $X_{1}$ and $X_{2}$, respectively. Since $X_{1} \perp X_{2}$ holds wrt $p$, by symmetry and decomposition, $\{u\} \cup Z_{2} \perp Z_{1}$ holds too. By symmetry and weak union, $\{u\} \perp Z_{1} \mid Z_{2}$ holds as well. Now since $Z_{1} \cup Z_{2}$ are the parents of $u$, according to the comment that follows Theorem 1, $\{u\} \perp Y \mid Z_{1} \cup Z_{2}$ holds, where $Y$ is the set of $u$ 's nondescendants except its parents. Consequently, by the contraction property, $\{u\} \perp Z_{1} \cup Y \mid Z_{2}$ holds. Since $Z_{2}$ is a proper subset of $Z_{1} \cup Z_{2}$ (because $Z_{1}$ contains $v$ ), where $Z_{1} \cup Z_{2}$ are the parents of $u$ in $D, D$ is not minimal.

Theorem 3: If two nodes are disconnected in some minimal Bayesian network of $p(U)$, then they are disconnected in every minimal Bayesian network of $p(U)$.

Proof: It suffices to show that any two minimal Bayesian networks of $p$ share the same maximal connected components. Let $D_{\mathrm{A}}$ and $D_{\mathrm{B}}$ be two minimal Bayesian networks of $p$. Let $C_{\mathrm{A}}$ and $C_{\mathrm{B}}$ be maximal connected components of $D_{\mathrm{A}}$ and $D_{\mathrm{B}}$, respectively. Let $A$ and $B$ be the nodes of $C_{\mathrm{A}}$ and $C_{\mathrm{B}}$, respectively. We show that either $A=B$ or $A \cap B=\emptyset$. This demonstration will complete the proof, because for an arbitrary maximal connected component $C_{\mathrm{A}}$ in $D_{\mathrm{A}}$ there must exist a maximal connected component in $D_{\mathrm{B}}$ that shares at least one node with $C_{\mathrm{A}}$. Thus, by the above claim, it must have exactly the same nodes as $C_{\mathrm{A}}$. Therefore, each maximal connected component of $D_{\mathrm{A}}$ shares the same nodes with exactly one maximal connected component of $D_{\mathrm{B}}$. Hence, $D_{\mathrm{A}}$ and $D_{\mathrm{B}}$ share the same maximal connected components.

Since $D_{\mathrm{A}}$ is a minimal Bayesian network of $p$ and $C_{\mathrm{A}}$ is a maximal connected component of $D_{\mathrm{A}}$, there is no trail between $A$ and $U \backslash A$ and so, by Theorem 1, $A \perp U \backslash A$ holds. Using symmetry and decomposition, $(A \cap B) \perp(B \backslash A)$ holds too. Thus, by Lemma 2 , for $C_{\mathrm{B}}$ to be a maximal connected component, either $A \cap B$ or $B \backslash A$ must be empty, lest $D_{\mathrm{B}}$ 
would not be minimal. Similarly, for $C_{\mathrm{A}}$ to be a maximal connected component, $A \cap B$ or $A \backslash B$ must be empty. Thus, either $A=B$ or $A \cap B=\emptyset$.

Theorem 4: Two variables $x$ and $y$ are disconnected in every minimal network of $p(U)$ iff there exists a partition $U_{x}, U_{y}$ of $U$ such that $U_{x} \perp U_{y}$ and $x \in U_{x}$ and $y \in U_{y}$.

Proof: Suppose $x$ and $y$ are disconnected in some minimal network $D$ of $p(U)$. Let $U_{x}$ be the variables connected to $x$ in $D$, and $U_{y}$ be the rest of the variables in $U$. Thus there is no trail between $U_{x}$ and $U_{y}$ and so, by Theorem 1, $U_{x} \perp U_{y}$ holds (wrt $p$ ).

Suppose there exists a partition $U_{x}, U_{y}$ of $U$ such that $x \in$ $U_{x}, y \in U_{y}$, and $U_{x} \perp U_{y}$ holds (wrt $p$ ). We show that in every minimal Bayesian network $D$ of $p$, nodes $x$ and $y$ do not reside in the same connected component. Assume, to the contrary, that $x$ and $y$ reside in the same maximal connected component of some minimal Bayesian network $D$ of $p$, and that $C$ are the nodes of that component. Now, $\left(U_{x} \cap C\right) \perp\left(U_{y} \cap C\right)$ holds (wrt $p$ ) because it follows from $U_{x} \perp U_{y}$ by the symmetry and decomposition properties. Moreover, $U_{x} \cap C$ and $U_{y} \cap C$ are not empty, because they include $x$ and $y$, respectively. Since $U_{x}$ and $U_{y}$ are disjoint, the two sets $U_{x} \cap C, U_{y} \cap C$ partition $C$. Therefore, by Lemma 2, $D$ cannot be minimal, contrary to our assumption.

\section{Alternative DEFINITIONS FOR RELATEDNESS}

In this section, we discuss several possibilities for defining probabilistic relatedness (or unrelatedness as a complementary notion), indicate the pitfalls of the proposed definitions and conclude with a definition that bypasses these pitfalls. The common ground of the proposed definitions is the idea that two variables are unrelated iff they are independent given an appropriately large set of contexts.

As a first alternative, we could define $a$ and $b$ to be unrelated [wrt $\left.p\left(u_{1}, \cdots, u_{n}\right)\right]$ if and only if $\{a\} \perp\{b\} \mid V=\mathbf{V}$ where $V$ is a subset of variables of $U \backslash\{a, b\}$ and $\mathbf{V}$ is a specific assignment to each variable in $V$. In other words, a context consists of a set of assignments to a subset of variables and $a$ and $b$ are unrelated iff they are independent given any such context. For example, if $U=\{a, b, c\}$ and if both $a \perp b$ and $a \perp b \mid c$ hold, then $a$ and $b$ are said to be unrelated. The following well-known property of conditional independence [1], [12], which we call property $\mathrm{B}$,

$$
\{a\} \perp\{b\} \text { and }\{a\} \perp\{b\} \mid\{c\} \Rightarrow\{a, c\} \perp\{b\} \text { or }\{a\} \perp\{b, c\}
$$

holds whenever $c$ is a binary variable. The converse of (7) follows immediately from (3)-(6). Thus, due to Theorem 4, we conclude that in any minimal network of $p(a, b, c)$, if $c$ is a binary variable, then $a$ and $b$ will reside on two distinct components iff they are unrelated (wrt $p$ ).

The technical problem with this definition lies in the fact that if $c$ is not a binary variable, then property B does not hold anymore. The difficulty can be traced to the fact that if we conceive $a$ and $b$ to be unrelated (and therefore expect $a$ and $b$ to be disconnected), we indeed mean to say that $a$ and $b$ are marginally independent and conditionally independent given any possible context. One particular context not considered in our first attempt is the situation when $c$ is equal to either $c_{i}$ or $c_{j}$ but we do not know to which value. Of course, if $c$ is a binary variable, then saying that $c$ gets one of its values is a tautology that adds nothing to our knowledge but if $c$ is not a binary variable, then restricting the domain of $c$ is a new context and so if $a$ and $b$ are to be considered unrelated, then they should also be independent conditioned on $c=c_{i} \vee c_{j}$.

Indeed the following theorem shows one way to extend property B to nonbinary variables. This theorem justifies a second definition of relatedness for the simple case of three variables.

Theorem 5: Let $p(a, b, c)$ be a joint probability distribution of three random variables $a, b$, and $c$. If $\{a\} \perp\{b\}$ and $\{a\} \perp\{b\} \mid c=c_{i}$ for $i=1, \cdots, k$ and if $\{a\} \perp\{b\} \mid c=c_{i} \vee c_{j}$ for every $i$ and $j, 1 \leq i<j \leq k$ (i.e., $a$ and $b$ are unrelated), then either $\{a, c\} \perp\{b\}$ or $\{a\} \perp\{b, c\}$.

Proof: Let $c_{i}$ be a value of $c$. We say that $c_{i}$ has a Type I factorization if $p\left(a, b, c_{i}\right)=p\left(a, c_{i}\right) p(b)$ and it has a Type II factorization if $p\left(a, b, c_{i}\right)=p(a) p\left(b, c_{i}\right)$. We shall now prove that every pair of values $c_{i_{1}}$ and $c_{i_{2}}$ of $c$ has a common factorization, namely, either both values have a Type I factorization or both have a Type II factorization. This observation completes the proof because it implies that all values of $c$ have a common factorization either of Type I or of Type II. Rearrange the values of $c$ such that $c_{i_{1}}$ is one value and the disjunction of all other values is considered to be the single second value. According to property $\mathrm{B}$, the value $c_{i_{1}}$ either has a Type I factorization or a Type II factorization. So is the case with $c_{i_{2}}$ as well. If both values have the same factorization, then the proof is completed. Suppose, without loss of generality, that $c_{i_{1}}$ has a Type I factorization and that $c_{i_{2}}$ has a Type II factorization. Now rearrange the values of $c$ such that $c_{i_{1}} \vee c_{i_{2}}$ is one value and the disjunction of the other values is the second value. According to property $\mathrm{B}$, the value $c_{i_{1}} \vee c_{i_{2}}$ has a Type I factorization or a Type II factorization. If it has a Type I factorization, and since $c_{i_{1}}$ has a Type I factorization, it follows, by subtracting the corresponding equations, that also $c_{i_{2}}$ must have a Type II factorization. Similarly, if $c_{i_{1}} \vee c_{i_{2}}$ has a Type II factorization then $c_{i_{1}}$ must have a Type II factorization as well.

A straightforward generalization of Theorem 5 to $n$ variables can be phrased as follows. For a pair of variables $a$ and $b$ in $U$, we define a new variable $c$ whose domain is the Cartesian product of the domains of the variables in $U \backslash\{a, b\}$. Now we say that $a$ and $b$ are unrelated wrt $p(U)$ if $\{a\} \perp\{b\}$, $\{a\} \perp\{b\} \mid\{c\}$ and $\{a\} \perp\{b\} \mid c=c_{i} \vee c_{j}$ for every two values of the combined variable $c$. The difficulty with this definition is that it is too strong. Due to Theorem 5, if $a$ and $b$ are unrelated wrt $p(U)$ according to this definition, then either $a$ is independent of $U \backslash\{a\}$ or $b$ is independent of $U \backslash\{b\}$. Consequently, either $a$ or $b$ is unrelated to all other variables and this claim is stronger than saying that $a$ and $b$ are unrelated merely between themselves.

So we conclude with the following definition.

Definition: Let $p\left(u_{1}, \cdots, u_{n}\right)$ be a probability distribution. Variables $u_{i}$ and $u_{j}$ are unrelated if $\left\{u_{i}\right\} \perp\left\{u_{j}\right\}$ and $\left\{u_{i}\right\} \perp\left\{u_{j}\right\} \mid\left\{v_{1}=V_{1}, \cdots, v_{m}=V_{m}\right\}$ for every disjunction 
of values $V_{1}, \cdots, V_{m}$ for $v_{1}, \cdots, v_{m}$, respectively, where $\left\{v_{1}, \cdots, v_{m}\right\}$ is an arbitrary subset of $\left\{u_{1}, \cdots, u_{n}\right\} \backslash$ $\left\{u_{i}, u_{j}\right\}$.

For example, suppose that $U=\{a, b, c, d\}$ and that the domains of $c$ and $d$ are $\left\{c_{1}, c_{2}\right\}$, and $\left\{d_{1}, d_{2}, d_{3}\right\}$, respectively. Then, according to our definition, $a$ and $b$ are unrelated (wrt $p$ ) iff $\{a\} \perp\{b\},\{a\} \perp\{b\} \mid c=c_{i}(i=1,2)$, $\{a\} \perp\{b\}\left|d=d_{j}(j=1, \cdots, 3),\{a\} \perp\{b\}\right| d=d_{j_{1}} \vee d_{j_{2}}(1 \leq$ $\left.j_{1}<j_{2} \leq 3\right),\{a\} \perp\{b\} \mid\left\{c=c_{i}, d=d_{j}\right\}(i=1,2, j=$ $1, \cdots, 3)$, and $\{a\} \perp\{b\} \mid\left\{c=c_{i}, d=d_{j_{1}} \vee d_{j_{2}}\right\}(i=$ $\left.1,2,1 \leq j_{1}<j_{2} \leq 3\right)$.

Note that when $u_{1}, \cdots, u_{n}$ are all binary variables, then the sets $V_{i}$ are singletons, namely, a single specific assignment for $v_{i}$. Therefore, for binary variables, our final definition coincides with our first one.

\section{Proof of Equivalence}

We now show that relatedness and connectedness are equivalent when all variables are binary and when the distribution $p$ is strictly positive.

Definition: A strictly positive binary distribution $P\left(u_{1}, \cdots, u_{n}\right)$ is a probability distribution where every variable has a domain of two values-say, true and false-and every combination of the variables' values has a probability greater than zero.

First we must generalize property B.

Theorem 6: Let $p\left(u_{1}, \cdots, u_{n}, e\right)$ be a strictly positive binary distribution. Let $\left\{X_{1}, X_{2}\right\},\left\{Y_{1}, Y_{2}\right\}$, and $\left\{Z_{1}, Z_{2}\right\}$ be three partitions of $U=\left\{u_{1}, \cdots, u_{n}\right\}$. Let $R_{1}$ be $X_{1} \cap Y_{1} \cap Z_{1}$, and $R_{2}$ be $X_{2} \cap Y_{2} \cap Z_{2}$. Then

$$
\begin{aligned}
& X_{1} \perp X_{2} \text { and } Y_{1} \perp Y_{2} \mid e=\mathbf{e}^{\prime} \text { and } Z_{1} \perp Z_{2} \mid e=\mathbf{e}^{\prime \prime} \\
& \quad \Rightarrow R_{1} \perp\left(\{e\} \cup U \backslash R_{1}\right) \text { or } R_{2} \perp\left(\{e\} \cup U \backslash R_{2}\right)
\end{aligned}
$$

where $\mathbf{e}^{\prime}$ and $\mathbf{e}^{\prime \prime}$ are two distinct values of $e$.

When all three partitions are identical, the above theorem can be phrased as follows. If two sets of variables $A$ and $B$ are marginally independent, and if $A \perp B \mid e$ holds as well, then either $A \perp(\{e\} \cup B)$ or $B \perp(\{e\} \cup A)$. This special case is precisely property $B$. The proof of Theorem 6 is given in the Appendix.

Theorem 7: Let $p\left(u_{1}, \cdots, u_{n}, u_{n+1}\right)$ be a strictly positive binary distribution. Suppose $x$ and $y$ are in $\left\{u_{1}, \cdots, u_{n+1}\right\}$. Then, $x$ and $y$ are unrelated (wrt $p$ ) if and only if $x$ and $y$ are disconnected in some minimal Bayesian network of $p$.

Proof: If $x$ and $y$ are disconnected in some minimal network, then according to Theorem 4 there exists a partition $U_{x}, U_{y}$ of $U$ such that $x \in U_{x}$ and $y \in U_{y}$ and $U_{x} \perp U_{y}$. Thus, using symmetry (3), decomposition (4) and weak union (5) it follows that $\{x\} \perp\{y\} \mid Z$ where $Z$ is an arbitrary subset of $U \backslash\{x, y\}$. Thus, $x$ and $y$ are unrelated wrt $(p)$.

The converse is proven by induction on $n$. If $n=1$, and $x$ and $y$ are unrelated, then $\{x\} \perp\{y\}$ holds wrt $p$. Consequently, there exists a partition $U_{x}=\{x\}, U_{y}=\{y\}$ of $U$ such that $U_{x} \perp U_{y}$ and so $x$ and $y$ are disconnected in any minimal network of $p$. Otherwise, assume without loss of generality that $x$ is $u_{1}$ and $y$ is $u_{2}$, and denote $u_{n+1}$ by $e$. Since $x$ and $y$ are unrelated wrt $p\left(u_{1}, \cdots, u_{n+1}\right), x$ and $y$ are also unrelated wrt $p\left(u_{1}, \cdots, u_{n}\right), p\left(u_{1}, \cdots, u_{n} \mid e=\mathbf{e}^{\prime}\right)$, and $p\left(u_{1}, \cdots, u_{n} \mid e=\right.$ $\mathbf{e}^{\prime \prime}$ ), where $\mathbf{e}^{\prime}$ and $\mathbf{e}^{\prime \prime}$ are two distinct values of $u_{n+1}$. Thus, by applying the induction hypothesis three times, we conclude that there are three partitions $\left\{X_{1}, X_{2}\right\},\left\{Y_{1}, Y_{2}\right\}$, and $\left\{Z_{1}, Z_{2}\right\}$ of $U=\left\{u_{1}, \cdots, u_{n}\right\}$ such that $x$ is in $X_{1}, Y_{1}$, and $Z_{1}$, and $y$ is in $X_{2}, Y_{2}$, and $Z_{2}$. Hence, the antecedents of (8) are satisfied. Consequently, $\left\{u_{1}, \cdots, u_{n+1}\right\}$ can be partitioned into two marginally independent sets: either $R_{1}$ and $U \backslash R_{1}$, or $R_{2}$ and $U \backslash R_{2}$, where $R_{1}$ is $X_{1} \cap Y_{1} \cap Z_{1}$ and $R_{2}$ is $X_{2} \cap Y_{2} \cap Z_{2}$. Because, in both cases, one set contains $x$ and the other contains $y$, it follows that $x$ and $y$ are disconnected.

\section{SUMMARY}

This paper shows that for strictly positive binary distributions the notion of probabilistic relatedness as defined herein is equivalent to the notion of connectedness in minimal Bayesian networks. We conjecture that the equivalence established holds also when these restrictions are lifted.

\section{APPENDIX}

Below, we prove Theorem 6. First, we phrase the theorem differently.

Theorem 8: Strictly positive binary distributions satisfy the following property: ${ }^{3}$

$$
\begin{aligned}
& \left(A_{1} A_{2} A_{3} A_{4} \perp B_{1} B_{2} B_{3} B_{4} \mid \emptyset\right) \\
& \&\left(A_{1} A_{2} B_{3} B_{4} \perp B_{1} B_{2} A_{3} A_{4} \mid e=\mathbf{e}^{\prime}\right) \\
& \&\left(A_{1} A_{3} B_{2} B_{4} \perp B_{1} B_{3} A_{2} A_{4} \mid e=\mathbf{e}^{\prime \prime}\right) \\
& \Rightarrow\left(A_{1} \perp e A_{2} A_{3} A_{4} B_{1} B_{2} B_{3} B_{4} \mid \emptyset\right) \\
& \vee\left(B_{1} \perp e A_{1} A_{2} A_{3} A_{4} B_{2} B_{3} B_{4} \mid \emptyset\right)
\end{aligned}
$$

where all sets mentioned are pairwise disjoint and do not contain $e$, and $\mathbf{e}^{\prime}$ and $\mathbf{e}^{\prime \prime}$ are distinct values of $e$.

To obtain the original theorem, we set $A_{1} A_{2} A_{3} A_{4}$, $B_{1} B_{2} B_{3} B_{4}, \quad A_{1} A_{2} B_{3} B_{4}, \quad B_{1} B_{2} A_{3} A_{4}, \quad A_{1} A_{3} B_{2} B_{4}$, and $B_{1} B_{3} A_{2} A_{4}$ to be equal to $X_{1}, X_{2}, Y_{1}, Y_{2}, Z_{1}$, and $Z_{2}$ of the original theorem, respectively.

Denote the three antecedents of (9) by $I_{1}, I_{2}$, and $I_{3}$. We need the following two Lemmas.

Lemma 9: Let $X$ and $Y$ be two disjoint sets of variables, and let $\mathbf{e}$ be an instance of a single binary variable $e$ not in $X \cup Y$. Let $p$ be a joint probability distribution of the variables $X \cup Y \cup\{e\}$. If $(X \perp Y \mid e=\mathbf{e})$ holds for $p$, then for every pair of instances $\mathbf{X}^{\prime}, \mathbf{X}^{\prime \prime}$ of $X$ and $\mathbf{Y}^{\prime}, \mathbf{Y}^{\prime \prime}$ of $Y$, the following equation must hold:

$$
\frac{p\left(\mathbf{e} \mid \mathbf{X}^{\prime} \mathbf{Y}^{\prime}\right) p\left(\mathbf{X}^{\prime} \mathbf{Y}^{\prime}\right)}{p\left(\mathbf{e} \mid \mathbf{X}^{\prime \prime} \mathbf{Y}^{\prime}\right) p\left(\mathbf{X}^{\prime \prime} \mathbf{Y}^{\prime}\right)}=\frac{p\left(\mathbf{e} \mid \mathbf{X}^{\prime} \mathbf{Y}^{\prime \prime}\right) p\left(\mathbf{X}^{\prime} \mathbf{Y}^{\prime \prime}\right)}{p\left(\mathbf{e} \mid \mathbf{X}^{\prime \prime} \mathbf{Y}^{\prime \prime}\right) p\left(\mathbf{X}^{\prime \prime} \mathbf{Y}^{\prime \prime}\right)}
$$

Proof: Bayes' theorem states that

$$
p\left(\mathbf{X}^{\prime} \mid \mathbf{e} \mathbf{Y}^{\prime}\right)=\frac{p\left(\mathbf{e} \mid \mathbf{X}^{\prime} \mathbf{Y}^{\prime}\right) p\left(\mathbf{X}^{\prime} \mathbf{Y}^{\prime}\right)}{p\left(\mathbf{e} \mathbf{Y}^{\prime}\right)}
$$

\footnotetext{
${ }^{3}$ In complicated expressions, $A_{1} A_{2}$ is used as a shorthand notation for $A_{1} \cup A_{2}$ and $e A_{1}$ denotes $\{e\} \cup A_{1}$.
} 
Thus,

$$
\begin{aligned}
\frac{p\left(\mathbf{e} \mid \mathbf{X}^{\prime} \mathbf{Y}^{\prime}\right) p\left(\mathbf{X}^{\prime} \mathbf{Y}^{\prime}\right)}{p\left(\mathbf{e} \mid \mathbf{X}^{\prime \prime} \mathbf{Y}^{\prime}\right) p\left(\mathbf{X}^{\prime \prime} \mathbf{Y}^{\prime}\right)} & =\frac{p\left(\mathbf{X}^{\prime} \mid \mathbf{e}, \mathbf{Y}^{\prime}\right)}{p\left(\mathbf{X}^{\prime \prime} \mid \mathbf{e}, \mathbf{Y}^{\prime}\right)}=\frac{p\left(\mathbf{X}^{\prime} \mid \mathbf{e}, \mathbf{Y}^{\prime \prime}\right)}{p\left(\mathbf{X}^{\prime \prime} \mid \mathbf{e}, \mathbf{Y}^{\prime \prime}\right)} \\
& =\frac{p\left(\mathbf{e} \mid \mathbf{X}^{\prime} \mathbf{Y}^{\prime \prime}\right) p\left(\mathbf{X}^{\prime} \mathbf{Y}^{\prime \prime}\right)}{p\left(\mathbf{e} \mid \mathbf{X}^{\prime \prime} \mathbf{Y}^{\prime \prime}\right) p\left(\mathbf{X}^{\prime \prime} \mathbf{Y}^{\prime \prime}\right)}
\end{aligned}
$$

The middle equality follows from the fact that $(X \perp Y \mid e=\mathbf{e})$ holds for $p$.

Lemma 10: Let $A_{1}, A_{2}, A_{3}, A_{4}, B_{1}, B_{2}, B_{3}$, and $B_{4}$ be disjoint sets of variables, and $e$ be a single binary variable not contained in any of these sets. Let $p$ be a joint probability distribution of the union of these variables. If the antecedents $I_{1}, I_{2}$, and $I_{3}$ of (9) hold for $p$, then the following conditions must also hold:

$$
\begin{aligned}
& \left(A_{1} \perp e \mid \mathbf{A}_{2}^{\prime} \mathbf{A}_{3}^{\prime} \mathbf{A}_{4}^{\prime} \mathbf{B}_{1}^{\prime} \mathbf{B}_{2}^{\prime} \mathbf{B}_{3}^{\prime} \mathbf{B}_{4}^{\prime}\right) \\
& \quad \Rightarrow\left(A_{1} \perp e \mid \mathbf{A}_{2}^{\prime} \mathbf{A}_{3}^{\prime} \mathbf{A}_{4}^{\prime} B_{1} B_{2} \mathbf{B}_{3}^{\prime} \mathbf{B}_{4}^{\prime}\right) \\
& \left(B_{1} \perp e \mid \mathbf{A}_{1}^{\prime} \mathbf{A}_{2}^{\prime} \mathbf{A}_{3}^{\prime} \mathbf{A}_{4}^{\prime} \mathbf{B}_{2}^{\prime} \mathbf{B}_{3}^{\prime} \mathbf{B}_{4}^{\prime}\right) \\
& \quad \Rightarrow\left(B_{1} \perp e \mid A_{1} A_{2} \mathbf{A}_{3}^{\prime} \mathbf{A}_{4}^{\prime} \mathbf{B}_{2}^{\prime} \mathbf{B}_{3}^{\prime} \mathbf{B}_{4}^{\prime}\right) \\
& \left(A_{1} \perp e \mid \mathbf{A}_{2}^{\prime} \mathbf{A}_{3}^{\prime} \mathbf{A}_{4}^{\prime} \mathbf{B}_{1}^{\prime} \mathbf{B}_{2}^{\prime} \mathbf{B}_{3}^{\prime} \mathbf{B}_{4}^{\prime}\right) \\
& \quad \Rightarrow\left(A_{1} \perp e \mid \mathbf{A}_{2}^{\prime} \mathbf{A}_{3}^{\prime} \mathbf{A}_{4}^{\prime} B_{1} \mathbf{B}_{2}^{\prime} B_{3} \mathbf{B}_{4}^{\prime}\right) \\
& \left(B_{1} \perp e \mid \mathbf{A}_{1}^{\prime} \mathbf{A}_{2}^{\prime} \mathbf{A}_{3}^{\prime} \mathbf{A}_{4}^{\prime} \mathbf{B}_{2}^{\prime} \mathbf{B}_{3}^{\prime} \mathbf{B}_{4}^{\prime}\right) \\
& \quad \Rightarrow\left(B_{1} \perp e \mid A_{1} \mathbf{A}_{2}^{\prime} A_{3} \mathbf{A}_{4}^{\prime} \mathbf{B}_{2}^{\prime} \mathbf{B}_{3}^{\prime} \mathbf{B}_{4}^{\prime}\right) \\
& \left(A_{1} \perp e \mid \mathbf{A}_{2}^{\prime} \mathbf{A}_{3}^{\prime} \mathbf{A}_{4}^{\prime} \mathbf{B}_{1}^{\prime} \mathbf{B}_{2}^{\prime} \mathbf{B}_{3}^{\prime} \mathbf{B}_{4}^{\prime}\right) \\
& \quad \Rightarrow\left(A_{1} \perp e \mid \mathbf{A}_{2}^{\prime} \mathbf{A}_{3}^{\prime} A_{4} B_{1} \mathbf{B}_{2}^{\prime} \mathbf{B}_{3}^{\prime} \mathbf{B}_{4}^{\prime}\right) \\
& \left(B_{1} \perp e \mid \mathbf{A}_{1}^{\prime} \mathbf{A}_{2}^{\prime} \mathbf{A}_{3}^{\prime} \mathbf{A}_{4}^{\prime} \mathbf{B}_{2}^{\prime} \mathbf{B}_{3}^{\prime} \mathbf{B}_{4}^{\prime}\right) \\
& \Rightarrow\left(B_{1} \perp e \mid A_{1} \mathbf{A}_{2}^{\prime} \mathbf{A}_{3}^{\prime} \mathbf{A}_{4}^{\prime} \mathbf{B}_{2}^{\prime} \mathbf{B}_{3}^{\prime} B_{4}\right)
\end{aligned}
$$

where each $\mathbf{A}_{i}^{\prime}$ and $\mathbf{B}_{i}^{\prime}$ denote a specific value for $A_{i}$ and $B_{i}$, respectively. [In words, (10) states that if $A_{1}$ and $e$ are conditionally independent for one specific value $\mathbf{B}_{1}^{\prime}$ of $B_{1}$ and $\mathbf{B}_{2}^{\prime}$ of $B_{2}$, then they are conditionally independent given every value of $B_{1}$ and $B_{2}$, provided the values of the other variables remain unaltered. The other five equations have a similar interpretation.]

Proof: First, we prove (10). Then we show that the proofs of (11)-(13) are symmetric. Finally, we will prove (14) and (15). Let $X=A_{1} A_{2} B_{3} B_{4}$ and $Y=B_{1} B_{2} A_{3} A_{4}$. Then, Lemma 9 and $I_{2}$ yield (15a), shown at the bottom of the page, where $\mathbf{A}_{1}^{*}, \mathbf{B}_{1}^{*}$, and $\mathbf{B}_{2}^{*}$ are arbitrary instances of $A_{1}, B_{1}$, and $B_{2}$, respectively. Applying $I_{1}$ and cancelling equal terms yields

$$
\begin{aligned}
& \frac{p\left(\mathbf{e} \mid \mathbf{A}_{1}^{\prime} \mathbf{A}_{2}^{\prime} \mathbf{A}_{3}^{\prime} \mathbf{A}_{4}^{\prime} \mathbf{B}_{1}^{\prime} \mathbf{B}_{2}^{\prime} \mathbf{B}_{3}^{\prime} \mathbf{B}_{4}^{\prime}\right)}{p\left(\mathbf{e} \mid \mathbf{A}_{1}^{*} \mathbf{A}_{2}^{\prime} \mathbf{A}_{3}^{\prime} \mathbf{A}_{4}^{\prime} \mathbf{B}_{1}^{\prime} \mathbf{B}_{2}^{\prime} \mathbf{B}_{3}^{\prime} \mathbf{B}_{4}^{\prime}\right)} \\
& \quad=\frac{p\left(\mathbf{e} \mid \mathbf{A}_{1}^{\prime} \mathbf{A}_{2}^{\prime} \mathbf{A}_{3}^{\prime} \mathbf{A}_{4}^{\prime} \mathbf{B}_{1}^{*} \mathbf{B}_{2}^{*} \mathbf{B}_{3}^{\prime} \mathbf{B}_{4}^{\prime}\right)}{p\left(\mathbf{e} \mid \mathbf{A}_{1}^{*} \mathbf{A}_{2}^{\prime} \mathbf{A}_{3}^{\prime} \mathbf{A}_{4}^{\prime} \mathbf{B}_{1}^{*} \mathbf{B}_{2}^{*} \mathbf{B}_{3}^{\prime} \mathbf{B}_{4}^{\prime}\right)} .
\end{aligned}
$$

Furthermore, $\left(A_{1} \perp e \mid \mathbf{A}_{2}^{\prime} \mathbf{A}_{3}^{\prime} \mathbf{A}_{4}^{\prime} \mathbf{B}_{1}^{\prime} \mathbf{B}_{2}^{\prime} \mathbf{B}_{3}^{\prime} \mathbf{B}_{4}^{\prime}\right)$ [the antecedent of (10)] implies that

$$
\begin{aligned}
& p\left(\mathbf{e} \mid \mathbf{A}_{1}^{\prime} \mathbf{A}_{2}^{\prime} \mathbf{A}_{3}^{\prime} \mathbf{A}_{4}^{\prime} \mathbf{B}_{1}^{\prime} \mathbf{B}_{2}^{\prime} \mathbf{B}_{3}^{\prime} \mathbf{B}_{4}^{\prime}\right) \\
& \quad=p\left(\mathbf{e} \mid \mathbf{A}_{1}^{*} \mathbf{A}_{2}^{\prime} \mathbf{A}_{3}^{\prime} \mathbf{A}_{4}^{\prime} \mathbf{B}_{1}^{\prime} \mathbf{B}_{2}^{\prime} \mathbf{B}_{3}^{\prime} \mathbf{B}_{4}^{\prime}\right) .
\end{aligned}
$$

Thus, from (16), it follows that

$$
\begin{aligned}
& p\left(\mathbf{e} \mid \mathbf{A}_{1}^{\prime} \mathbf{A}_{2}^{\prime} \mathbf{A}_{3}^{\prime} \mathbf{A}_{4}^{\prime} \mathbf{B}_{1}^{*} \mathbf{B}_{2}^{*} \mathbf{B}_{3}^{\prime} \mathbf{B}_{4}^{\prime}\right) \\
& \quad=p\left(\mathbf{e} \mid \mathbf{A}_{1}^{*} \mathbf{A}_{2}^{\prime} \mathbf{A}_{3}^{\prime} \mathbf{A}_{4}^{\prime} \mathbf{B}_{1}^{*} \mathbf{B}_{2}^{*} \mathbf{B}_{3}^{\prime} \mathbf{B}_{4}^{\prime}\right) .
\end{aligned}
$$

Subtracting each side of (17) from 1 yields

$$
\begin{aligned}
& p\left(\overline{\mathbf{e}} \mid \mathbf{A}_{1}^{\prime} \mathbf{A}_{2}^{\prime} \mathbf{A}_{3}^{\prime} \mathbf{A}_{4}^{\prime} \mathbf{B}_{1}^{*} \mathbf{B}_{2}^{*} \mathbf{B}_{3}^{\prime} \mathbf{B}_{4}^{\prime}\right) \\
& \quad=p\left(\overline{\mathbf{e}} \mid \mathbf{A}_{1}^{*} \mathbf{A}_{2}^{\prime} \mathbf{A}_{3}^{\prime} \mathbf{A}_{4}^{\prime} \mathbf{B}_{1}^{*} \mathbf{B}_{2}^{*} \mathbf{B}_{3}^{\prime} \mathbf{B}_{4}^{\prime}\right) .
\end{aligned}
$$

Thus, $\left(A_{1} \perp e \mid \mathbf{A}_{2}^{\prime} \mathbf{A}_{3}^{\prime} \mathbf{A}_{4}^{\prime} \mathbf{B}_{1}^{*} \mathbf{B}_{2}^{*} \mathbf{B}_{3}^{\prime} \mathbf{B}_{4}^{\prime}\right)$ holds for $p$. Because $\mathbf{B}_{1}^{*}$ and $\mathbf{B}_{2}^{*}$ are arbitrary instances, $\left(A_{1} \perp e \mid \mathbf{A}_{2}^{\prime} \mathbf{A}_{3}^{\prime} \mathbf{A}_{4}^{\prime} B_{1} B_{2} \mathbf{B}_{3}^{\prime} \mathbf{B}_{4}^{\prime}\right)$ also holds for $p$. Thus, (10) is proved.

Equation (11) is symmetric with respect to (10) by switching the role of $A_{1}$ with that of $B_{1}$ and the role of $A_{2}$ with that of $B_{2}$. Equation (12) is symmetric with respect to (10) by switching the roles of $B_{2}$ and $B_{3}$. Equation (13) is symmetric with respect to (11) by switching the roles of $A_{2}$ and $A_{3}$.

Now we prove (14). Equation (15) is symmetric with respect to (14) by switching the role of $A_{1}$ with that of $B_{1}$ and the role of $A_{4}$ with that of $B_{4}$.

Let $X=A_{1} A_{2} B_{3} B_{4}$ and $Y=B_{1} B_{2} A_{3} A_{4}$. Applying Lemma 9 and $I_{2}$ and then using $I_{1}$ to cancel equal terms, yields the following equation:

$$
\begin{aligned}
& \frac{p\left(\mathbf{e} \mid \mathbf{A}_{1}^{\prime} \mathbf{A}_{2}^{\prime} \mathbf{A}_{3}^{\prime} \mathbf{A}_{4}^{\prime} \mathbf{B}_{1}^{\prime} \mathbf{B}_{2}^{\prime} \mathbf{B}_{3}^{\prime} \mathbf{B}_{4}^{\prime}\right) p\left(\mathbf{A}_{1}^{\prime} \mathbf{A}_{2}^{\prime} \mathbf{A}_{3}^{\prime} \mathbf{A}_{4}^{\prime}\right)}{p\left(\mathbf{e} \mid \mathbf{A}_{1}^{*} \mathbf{A}_{2}^{\prime} \mathbf{A}_{3}^{\prime} \mathbf{A}_{4}^{\prime} \mathbf{B}_{1}^{\prime} \mathbf{B}_{2}^{\prime} \mathbf{B}_{3}^{\prime} \mathbf{B}_{4}^{\prime}\right) p\left(\mathbf{A}_{1}^{*} \mathbf{A}_{2}^{\prime} \mathbf{A}_{3}^{\prime} \mathbf{A}_{4}^{\prime}\right)} \\
& =\frac{p\left(\mathbf{e} \mid \mathbf{A}_{1}^{\prime} \mathbf{A}_{2}^{\prime} \mathbf{A}_{3}^{\prime} \mathbf{A}_{4}^{*} \mathbf{B}_{1}^{*} \mathbf{B}_{2}^{\prime} \mathbf{B}_{3}^{\prime} \mathbf{B}_{4}^{\prime}\right) p\left(\mathbf{A}_{1}^{\prime} \mathbf{A}_{2}^{\prime} \mathbf{A}_{3}^{\prime} \mathbf{A}_{4}^{*}\right)}{p\left(\mathbf{e} \mid \mathbf{A}_{1}^{*} \mathbf{A}_{2}^{\prime} \mathbf{A}_{3}^{\prime} \mathbf{A}_{4}^{*} \mathbf{B}_{1}^{*} \mathbf{B}_{2}^{\prime} \mathbf{B}_{3}^{\prime} \mathbf{B}_{4}^{\prime}\right) p\left(\mathbf{A}_{1}^{*} \mathbf{A}_{2}^{\prime} \mathbf{A}_{3}^{\prime} \mathbf{A}_{4}^{*}\right)}
\end{aligned}
$$

where $\mathbf{A}_{1}^{*}, \mathbf{B}_{1}^{*}$, and $\mathbf{A}_{4}^{*}$ are arbitrary instances of $A_{1}, B_{1}$, and $A_{4}$, respectively. Similarly, let $X=A_{1} A_{3} B_{2} B_{4}$ and $Y=B_{1} B_{3} A_{2} A_{4}$. Then, applying Lemma 9 and $I_{3}$ and using $I_{1}$ to cancel equal terms, yields the following equation:

$$
\begin{aligned}
& \frac{p\left(\overline{\mathbf{e}} \mid \mathbf{A}_{1}^{\prime} \mathbf{A}_{2}^{\prime} \mathbf{A}_{3}^{\prime} \mathbf{A}_{4}^{\prime} \mathbf{B}_{1}^{\prime} \mathbf{B}_{2}^{\prime} \mathbf{B}_{3}^{\prime} \mathbf{B}_{4}^{\prime}\right) p\left(\mathbf{A}_{1}^{\prime} \mathbf{A}_{2}^{\prime} \mathbf{A}_{3}^{\prime} \mathbf{A}_{4}^{\prime}\right)}{p\left(\overline{\mathbf{e}} \mid \mathbf{A}_{1}^{*} \mathbf{A}_{2}^{\prime} \mathbf{A}_{3}^{\prime} \mathbf{A}_{4}^{\prime} \mathbf{B}_{1}^{\prime} \mathbf{B}_{2}^{\prime} \mathbf{B}_{3}^{\prime} \mathbf{B}_{4}^{\prime}\right) p\left(\mathbf{A}_{1}^{*} \mathbf{A}_{2}^{\prime} \mathbf{A}_{3}^{\prime} \mathbf{A}_{4}^{\prime}\right)} \\
& \quad=\frac{p\left(\overline{\mathbf{e}} \mid \mathbf{A}_{1}^{\prime} \mathbf{A}_{2}^{\prime} \mathbf{A}_{3}^{\prime} \mathbf{A}_{4}^{*} \mathbf{B}_{1}^{*} \mathbf{B}_{2}^{\prime} \mathbf{B}_{3}^{\prime} \mathbf{B}_{4}^{\prime}\right) p\left(\mathbf{A}_{1}^{\prime} \mathbf{A}_{2}^{\prime} \mathbf{A}_{3}^{\prime} \mathbf{A}_{4}^{*}\right)}{p\left(\overline{\mathbf{e}} \mid \mathbf{A}_{1}^{*} \mathbf{A}_{2}^{\prime} \mathbf{A}_{3}^{\prime} \mathbf{A}_{4}^{*} \mathbf{B}_{1}^{*} \mathbf{B}_{2}^{\prime} \mathbf{B}_{3}^{\prime} \mathbf{B}_{4}^{\prime}\right) p\left(\mathbf{A}_{1}^{*} \mathbf{A}_{2}^{\prime} \mathbf{A}_{3}^{\prime} \mathbf{A}_{4}^{*}\right)} .
\end{aligned}
$$

Now $\left(A_{1} \perp e \mid \mathbf{A}_{2}^{\prime} \mathbf{A}_{3}^{\prime} \mathbf{A}_{4}^{\prime} \mathbf{B}_{1}^{\prime} \mathbf{B}_{2}^{\prime} \mathbf{B}_{3}^{\prime} \mathbf{B}_{4}^{\prime}\right)$ implies the following two conditions:

$$
\begin{aligned}
& p\left(\mathbf{e} \mid \mathbf{A}_{1}^{\prime} \mathbf{A}_{2}^{\prime} \mathbf{A}_{3}^{\prime} \mathbf{A}_{4}^{\prime} \mathbf{B}_{1}^{\prime} \mathbf{B}_{2}^{\prime} \mathbf{B}_{3}^{\prime} \mathbf{B}_{4}^{\prime}\right) \\
& \quad=p\left(\mathbf{e} \mid \mathbf{A}_{1}^{*} \mathbf{A}_{2}^{\prime} \mathbf{A}_{3}^{\prime} \mathbf{A}_{4}^{\prime} \mathbf{B}_{1}^{\prime} \mathbf{B}_{2}^{\prime} \mathbf{B}_{3}^{\prime} \mathbf{B}_{4}^{\prime}\right) \\
& p\left(\overline{\mathbf{e}} \mid \mathbf{A}_{1}^{\prime} \mathbf{A}_{2}^{\prime} \mathbf{A}_{3}^{\prime} \mathbf{A}_{4}^{\prime} \mathbf{B}_{1}^{\prime} \mathbf{B}_{2}^{\prime} \mathbf{B}_{3}^{\prime} \mathbf{B}_{4}^{\prime}\right) \\
& \quad=p\left(\overline{\mathbf{e}} \mid \mathbf{A}_{1}^{*} \mathbf{A}_{2}^{\prime} \mathbf{A}_{3}^{\prime} \mathbf{A}_{4}^{\prime} \mathbf{B}_{1}^{\prime} \mathbf{B}_{2}^{\prime} \mathbf{B}_{3}^{\prime} \mathbf{B}_{4}^{\prime}\right) .
\end{aligned}
$$

$$
\begin{aligned}
& \frac{p\left(\mathbf{e} \mid \mathbf{A}_{1}^{\prime} \mathbf{A}_{2}^{\prime} \mathbf{A}_{3}^{\prime} \mathbf{A}_{4}^{\prime} \mathbf{B}_{1}^{\prime} \mathbf{B}_{2}^{\prime} \mathbf{B}_{3}^{\prime} \mathbf{B}_{4}^{\prime}\right) p\left(\mathbf{A}_{1}^{\prime} \mathbf{A}_{2}^{\prime} \mathbf{A}_{3}^{\prime} \mathbf{A}_{4}^{\prime} \mathbf{B}_{1}^{\prime} \mathbf{B}_{2}^{\prime} \mathbf{B}_{3}^{\prime} \mathbf{B}_{4}^{\prime}\right)}{p\left(\mathbf{e} \mid \mathbf{A}_{1}^{*} \mathbf{A}_{2}^{\prime} \mathbf{A}_{3}^{\prime} \mathbf{A}_{4}^{\prime} \mathbf{B}_{1}^{\prime} \mathbf{B}_{2}^{\prime} \mathbf{B}_{3}^{\prime} \mathbf{B}_{4}^{\prime}\right) p\left(\mathbf{A}_{1}^{*} \mathbf{A}_{2}^{\prime} \mathbf{A}_{3}^{\prime} \mathbf{A}_{4}^{\prime} \mathbf{B}_{1}^{\prime} \mathbf{B}_{2}^{\prime} \mathbf{B}_{3}^{\prime} \mathbf{B}_{4}^{\prime}\right)} \\
& \quad=\frac{p\left(\mathbf{e} \mid \mathbf{A}_{1}^{\prime} \mathbf{A}_{2}^{\prime} \mathbf{A}_{3}^{\prime} \mathbf{A}_{4}^{\prime} \mathbf{B}_{1}^{*} \mathbf{B}_{2}^{*} \mathbf{B}_{3}^{\prime} \mathbf{B}_{4}^{\prime}\right) p\left(\mathbf{A}_{1}^{\prime} \mathbf{A}_{2}^{\prime} \mathbf{A}_{3}^{\prime} \mathbf{A}_{4}^{\prime} \mathbf{B}_{1}^{*} \mathbf{B}_{2}^{*} \mathbf{B}_{3}^{\prime} \mathbf{B}_{4}^{\prime}\right)}{p\left(\mathbf{e} \mid \mathbf{A}_{1}^{*} \mathbf{A}_{2}^{\prime} \mathbf{A}_{3}^{\prime} \mathbf{A}_{4}^{\prime} \mathbf{B}_{1}^{*} \mathbf{B}_{2}^{*} \mathbf{B}_{3}^{\prime} \mathbf{B}_{4}^{\prime}\right) p\left(\mathbf{A}_{1}^{*} \mathbf{A}_{2}^{\prime} \mathbf{A}_{3}^{\prime} \mathbf{A}_{4}^{\prime} \mathbf{B}_{1}^{*} \mathbf{B}_{2}^{*} \mathbf{B}_{3}^{\prime} \mathbf{B}_{4}^{\prime}\right)}
\end{aligned}
$$


After using (21) to cancel equal terms in (19) and using (22) to cancel equal terms in (20), we compare (19) and (20) and obtain

$$
\begin{aligned}
& \frac{p\left(\mathbf{e} \mid \mathbf{A}_{1}^{\prime} \mathbf{A}_{2}^{\prime} \mathbf{A}_{3}^{\prime} \mathbf{A}_{4}^{*} \mathbf{B}_{1}^{*} \mathbf{B}_{2}^{\prime} \mathbf{B}_{3}^{\prime} \mathbf{B}_{4}^{\prime}\right)}{p\left(\mathbf{e} \mid \mathbf{A}_{1}^{*} \mathbf{A}_{2}^{\prime} \mathbf{A}_{3}^{\prime} \mathbf{A}_{4}^{*} \mathbf{B}_{1}^{*} \mathbf{B}_{2}^{\prime} \mathbf{B}_{3}^{\prime} \mathbf{B}_{4}^{\prime}\right)} \\
& \quad=\frac{p\left(\mathbf{\mathbf { e }} \mid \mathbf{A}_{1}^{\prime} \mathbf{A}_{2}^{\prime} \mathbf{A}_{3}^{\prime} \mathbf{A}_{4}^{*} \mathbf{B}_{1}^{*} \mathbf{B}_{2}^{\prime} \mathbf{B}_{3}^{\prime} \mathbf{B}_{4}^{\prime}\right)}{p\left(\overline{\mathbf{e}} \mid \mathbf{A}_{1}^{*} \mathbf{A}_{2}^{\prime} \mathbf{A}_{3}^{\prime} \mathbf{A}_{4}^{*} \mathbf{B}_{1}^{*} \mathbf{B}_{2}^{\prime} \mathbf{B}_{3}^{\prime} \mathbf{B}_{4}^{\prime}\right)} .
\end{aligned}
$$

Equation (23) has the form

$$
\frac{x}{y}=\frac{1-x}{1-y}
$$

which yields $x=y$.

Consequently, we obtain $\left(A_{1} \perp e \mid \mathbf{A}_{2}^{\prime} \mathbf{A}_{3}^{\prime} \mathbf{A}_{4}^{*} \mathbf{B}_{1}^{*} \mathbf{B}_{2}^{\prime} \mathbf{B}_{3}^{\prime} \mathbf{B}_{4}^{\prime}\right)$. Furthermore, because $\mathbf{A}_{4}^{*}$ and $\mathbf{B}_{1}^{*}$ are arbitrary instances, $\left(A_{1} \perp e \mid \mathbf{A}_{2}^{\prime} \mathbf{A}_{3}^{\prime} A_{4} B_{1} \mathbf{B}_{2}^{\prime} \mathbf{B}_{3}^{\prime} \mathbf{B}_{4}^{\prime}\right)$ holds for $p$.

Next, we prove Theorem 6 . Let $C=A_{2} A_{3} A_{4}$ and $D=$ $B_{2} B_{3} B_{4}$. We will see that $I_{1}, I_{2}$, and $I_{3}$ imply the following four properties:

$$
\begin{gathered}
\left(A_{1} \perp e \mid C D B_{1}\right) \text { or }\left(B_{1} \perp e \mid C D A_{1}\right) \\
\left(A_{1} \perp e \mid C D B_{1}\right) \Rightarrow\left(A_{1} \perp A_{4} \mid A_{2} A_{3}\right) \\
\left(A_{1} \perp e \mid C D B_{1}\right) \&\left(A_{1} \perp A_{4} \mid A_{2} A_{3}\right) \Rightarrow\left(A_{1} \perp A_{3} \mid A_{2}\right) \\
\left(A_{1} \perp e \mid C D B_{1}\right) \&\left(A_{1} \perp A_{4} \mid A_{2} A_{3}\right) \&\left(A_{1} \perp A_{3} \mid A_{2}\right) \\
\Rightarrow\left(A_{1} \perp A_{2} \mid \emptyset\right) .
\end{gathered}
$$

First, we prove (9), using these four properties. Then, we will show that these properties are valid. From (24), there are two symmetric cases to consider. Without loss of generality, assume $\left(A_{1} \perp e \mid C D B_{1}\right)$ holds. [Otherwise, we switch the roles of subscripted $A$ 's with subscripted $B$ 's in (25)-(27).] By a single application of each of (25)-(27), the following independence statements are proved to hold for $p$ :

$$
\left(A_{1} \perp A_{2} \mid \emptyset\right),\left(A_{1} \perp A_{3} \mid A_{2}\right),\left(A_{1} \perp A_{4} \mid A_{2} A_{3}\right) .
$$

These three statements yield $\left(A_{1} \perp A_{2} A_{3} A_{4} \mid \emptyset\right)\left(\equiv I_{4}\right)$ by two applications of contraction. Consider (9). The statement $\left(A_{1} A_{2} A_{3} A_{4} \perp B_{1} B_{2} B_{3} B_{4} \mid \emptyset\right) \quad$ (i.e., $\left.\quad I_{1}\right)$ implies $\left(A_{1} \perp B_{1} B_{2} B_{3} B_{4} \mid A_{2} A_{3} A_{4}\right)$ using weak union, which together with $I_{4}$ imply using contraction $\left(A_{1} \perp A_{2} A_{3} A_{4} B_{1} B_{2} B_{3} B_{4} \mid \emptyset\right)$. This statement together with the statement $\left(A_{1} \perp e \mid C B_{1} D\right)$ imply, using contraction, the statement $\left(A_{1} \perp e A_{2} A_{3} A_{4} B_{1} B_{2} B_{3} B_{4} \mid \emptyset\right)$, thus completing the proof.

It remains to prove (24)-(27). First, we prove (24). Let $\mathbf{A}^{\prime}$, $\mathbf{A}^{\prime \prime}, \mathbf{B}^{\prime}, \mathbf{B}^{\prime \prime}, \mathbf{C}^{*}$, and $\mathbf{D}^{*}$ be arbitrary instances of $A_{1}, B_{1}$, $C$, and $D$, respectively. Let $X=A C$ and $Y=B D$. Then, Lemma 9 and $I_{2}$ yield the following equation:

$$
\begin{aligned}
& \frac{p\left(\mathbf{e} \mid \mathbf{A}^{\prime} \mathbf{C}^{*} \mathbf{D}^{*} \mathbf{B}^{\prime}\right) p\left(\mathbf{A}^{\prime} \mathbf{C}^{*} \mathbf{D}^{*} \mathbf{B}^{\prime}\right)}{p\left(\mathbf{e} \mid \mathbf{A}^{\prime \prime} \mathbf{C}^{*} \mathbf{D}^{*} \mathbf{B}^{\prime}\right) p\left(\mathbf{A}^{\prime \prime} \mathbf{C}^{*} \mathbf{D}^{*} \mathbf{B}^{\prime}\right)} \\
& \quad=\frac{p\left(\mathbf{e} \mid \mathbf{A}^{\prime} \mathbf{C}^{*} \mathbf{D}^{*} \mathbf{B}^{\prime \prime}\right) p\left(\mathbf{A}^{\prime} \mathbf{C}^{*} \mathbf{D}^{*} \mathbf{B}^{\prime \prime}\right)}{p\left(\mathbf{e} \mid \mathbf{A}^{\prime \prime} \mathbf{C}^{*} \mathbf{D}^{*} \mathbf{B}^{\prime \prime}\right) p\left(\mathbf{A}^{\prime \prime} \mathbf{C}^{*} \mathbf{D}^{*} \mathbf{B}^{\prime \prime}\right)} .
\end{aligned}
$$

From $I_{1}$, we obtain $p(A C D B)=p(A C) p(D B)$. Consequently, (28) yields

$$
\frac{p\left(\mathbf{e} \mid \mathbf{A}^{\prime} \mathbf{B}^{\prime} \mathbf{C}^{*} \mathbf{D}^{*}\right)}{p\left(\mathbf{e} \mid \mathbf{A}^{\prime \prime} \mathbf{B}^{\prime} \mathbf{C}^{*} \mathbf{D}^{*}\right)}=\frac{p\left(\mathbf{e} \mid \mathbf{A}^{\prime} \mathbf{B}^{\prime \prime} \mathbf{C}^{*} \mathbf{D}^{*}\right)}{p\left(\mathbf{e} \mid \mathbf{A}^{\prime \prime} \mathbf{B}^{\prime \prime} \mathbf{C}^{*} \mathbf{D}^{*}\right)} .
$$

Equation (29) has the following algebraic form, where subscripted $X \mathrm{~s}$ replace the corresponding terms

$$
\frac{X_{\mathbf{A}^{\prime} \mathbf{B}^{\prime}}}{X_{\mathbf{A}^{\prime \prime} \mathbf{B}^{\prime}}}=\frac{X_{\mathbf{A}^{\prime} \mathbf{B}^{\prime \prime}}}{X_{\mathbf{A}^{\prime \prime} \mathbf{B}^{\prime \prime}}} \text {. }
$$

Using Lemma 9 and $I_{3}$, we obtain a relationship similar to (29), where the only change is that $\mathbf{e}$ is replaced with $\overline{\mathbf{e}}$

$$
\frac{p\left(\overline{\mathbf{e}} \mid \mathbf{A}^{\prime} \mathbf{B}^{\prime} \mathbf{C}^{*} \mathbf{D}^{*}\right)}{p\left(\overline{\mathbf{e}} \mid \mathbf{A}^{\prime \prime} \mathbf{B}^{\prime} \mathbf{C}^{*} \mathbf{D}^{*}\right)}=\frac{p\left(\overline{\mathbf{e}} \mid \mathbf{A}^{\prime} \mathbf{B}^{\prime \prime} \mathbf{C}^{*} \mathbf{D}^{*}\right)}{p\left(\overline{\mathbf{e}} \mid \mathbf{A}^{\prime \prime} \mathbf{B}^{\prime \prime} \mathbf{C}^{*} \mathbf{D}^{*}\right)} .
$$

We rewrite (31) in terms of $X \mathrm{~s}$, and then use (30) to obtain

$$
\frac{1-X_{\mathbf{A}^{\prime} \mathbf{B}^{\prime}}}{1-X_{\mathbf{A}^{\prime \prime} \mathbf{B}^{\prime}}}=\frac{1-k X_{\mathbf{A}^{\prime} \mathbf{B}^{\prime}}}{1-k X_{\mathbf{A}^{\prime \prime} \mathbf{B}^{\prime}}}
$$

where $k=\left(X_{\mathbf{A}^{\prime} \mathbf{B}^{\prime \prime}} / X_{\mathbf{A}^{\prime} \mathbf{B}^{\prime}}\right)$. Equation (32) implies that either $X_{\mathbf{A}^{\prime} \mathbf{B}^{\prime \prime}}=X_{\mathbf{A}^{\prime} \mathbf{B}^{\prime}}$ (i.e., $k=1$ ) or $X_{\mathbf{A}^{\prime} \mathbf{B}^{\prime}}=X_{\mathbf{A}^{\prime \prime} \mathbf{B}^{\prime}}$. Because the choice of instances for $A_{1}$ and $B_{1}$ is arbitrary, at least one of the following two sequences of equalities must hold

- for every instance $\mathbf{B}$ of $B_{1}, X_{\mathbf{A}^{1} \mathbf{B}}=X_{\mathbf{A}^{2} \mathbf{B}}=\cdots=$ $X_{\mathbf{A}^{m} \mathbf{B}}$

- for every instance $\mathbf{A}$ of $A_{1}, X_{\mathbf{A B}^{1}}=X_{\mathbf{A B}^{2}}=\cdots=$ $X_{\mathrm{AB}^{n}}$;

where $\mathbf{A}^{1}, \cdots, A^{m}$ are the instances of $A_{1}$ and $\mathbf{B}^{1}, \cdots, \mathbf{B}^{n}$ are the instances of $B_{1}$.

Thus, by definition of the $X \mathrm{~s}$, we obtain

$$
\begin{aligned}
& \forall \mathbf{C}^{*} \mathbf{D}^{*} \text { instances of } C D\left[\left(e \perp A_{1} \mid \mathbf{C}^{*} \mathbf{D}^{*} B_{1}\right)\right. \\
& \left.\quad \text { or }\left(e \perp B_{1} \mid \mathbf{C}^{*} \mathbf{D}^{*} A_{1}\right)\right] .
\end{aligned}
$$

On the other hand, (24), which we are now proving, states

$$
\begin{aligned}
& {\left[\forall \mathbf{C}^{*} \mathbf{D}^{*}\left(e \perp A_{1} \mid \mathbf{C}^{*} \mathbf{D}^{*} B_{1}\right)\right]} \\
& \quad \text { or }\left[\forall \mathbf{C}^{*} \mathbf{D}^{*}\left(e \perp B_{1} \mid \mathbf{C}^{*} \mathbf{D}^{*} A_{1}\right)\right]
\end{aligned}
$$

which is stronger than (33). Equation (24) can also be written as follows:

$$
\neg\left(B_{1} \perp e \mid C D A_{1}\right) \Rightarrow\left(A_{1} \perp e \mid C D B_{1}\right) .
$$

We prove (35). The statement $\neg\left(B_{1} \perp e \mid C D A_{1}\right)$ implies that there exists instances $\mathbf{A}_{1}^{\prime}, \mathbf{A}_{2}^{\prime}, \mathbf{A}_{3}^{\prime}, \mathbf{A}_{4}^{\prime}, \mathbf{B}_{1}^{\prime}, \mathbf{B}_{2}^{\prime}, \mathbf{B}_{3}^{\prime}, \mathbf{B}_{4}^{\prime}$, and $\mathbf{e}^{\prime}$ of $A_{1}, A_{2}, A_{3}, A_{4}, B_{1}, B_{2}, B_{2}, B_{3}, B_{4}$, and $e$, respectively, such that

$$
\neg\left(\mathbf{B}_{1}^{\prime} \perp \mathbf{e}^{\prime} \mid \mathbf{A}_{1}^{\prime} \mathbf{A}_{2}^{\prime} \mathbf{A}_{3}^{\prime} \mathbf{A}_{4}^{\prime} \mathbf{B}_{2}^{\prime} \mathbf{B}_{3}^{\prime} \mathbf{B}_{4}^{\prime}\right) .
$$

Hence,

$$
\neg\left(B_{1} \perp e \mid A_{1} A_{2} \mathbf{A}_{3}^{\prime} \mathbf{A}_{4}^{\prime} \mathbf{B}_{2}^{\prime} \mathbf{B}_{3}^{\prime} \mathbf{B}_{4}^{\prime}\right) .
$$

From Lemma 10 [contrapositive form of (11)], (37) implies

$$
\neg\left(B_{1} \perp e \mid \mathbf{A}_{1}^{*} \mathbf{A}_{2}^{*} \mathbf{A}_{3}^{\prime} \mathbf{A}_{4}^{\prime} \mathbf{B}_{2}^{\prime} \mathbf{B}_{3}^{\prime} \mathbf{B}_{4}^{\prime}\right)
$$

where $\mathbf{A}_{1}^{*}$ and $\mathbf{A}_{2}^{*}$ are arbitrary instances of $A_{1}$ and $A_{2}$, respectively. Hence, in particular, if $\mathbf{A}_{1}^{*}=\mathbf{A}_{1}^{\prime}$, we have

$$
\neg\left(B_{1} \perp e \mid \mathbf{A}_{1}^{\prime} \mathbf{A}_{2}^{*} \mathbf{A}_{3}^{\prime} \mathbf{A}_{4}^{\prime} \mathbf{B}_{2}^{\prime} \mathbf{B}_{3}^{\prime} \mathbf{B}_{4}^{\prime}\right) .
$$

Similarly, from Lemma 10 (13), (39) implies

$$
\neg\left(B_{1} \perp e \mid \mathbf{A}_{1}^{*} \mathbf{A}_{2}^{*} \mathbf{A}_{3}^{*} \mathbf{A}_{4}^{\prime} \mathbf{B}_{2}^{\prime} \mathbf{B}_{3}^{\prime} \mathbf{B}_{4}^{\prime}\right)
$$


where $\mathbf{A}_{3}^{*}$ is an arbitrary instance of $A_{3}$. Also, from Lemma 10 (15), (40) implies

$$
\neg\left(B_{1} \perp e \mid \mathbf{A}_{1}^{*} \mathbf{A}_{2}^{*} \mathbf{A}_{3}^{*} \mathbf{A}_{4}^{\prime} \mathbf{B}_{2}^{\prime} \mathbf{B}_{3}^{\prime} \mathbf{B}_{4}^{*}\right)
$$

where $\mathbf{B}_{4}^{*}$ is an arbitrary instance of $B_{4}$. Examine (33). Equation (41) states that the second disjunct cannot be true for every instance of $A_{1} A_{2} A_{3} B_{1} B_{4}$ and $e$. Hence, for each of these instances the other disjunct must hold. That is,

$$
\forall \mathbf{A}_{1}^{*} \mathbf{A}_{2}^{*} \mathbf{A}_{3}^{*} \mathbf{B}_{1}^{*} \mathbf{B}_{4}^{*} \mathbf{e}^{*}\left(\mathbf{A}_{1}^{*} \perp \mathbf{e}^{*} \mid \mathbf{B}_{1}^{*} \mathbf{A}_{2}^{*} \mathbf{A}_{3}^{*} \mathbf{A}_{4}^{\prime} \mathbf{B}_{2}^{\prime} \mathbf{B}_{3}^{\prime} \mathbf{B}_{4}^{*}\right)
$$

or, equivalently,

$$
\left(A_{1} \perp e \mid B_{1} A_{2} A_{3} \mathbf{A}_{4}^{\prime} \mathbf{B}_{2}^{\prime} \mathbf{B}_{3}^{\prime} B_{4}\right) .
$$

Applying (43) to (10) yields,

$$
\left(A_{1} \perp e \mid B_{1} A_{2} A_{3} \mathbf{A}_{4}^{\prime} B_{2} \mathbf{B}_{3}^{\prime} B_{4}\right) .
$$

Similarly, applying (44) to (12) and (14) yields the statement

$$
\left(A_{1} \perp e \mid A_{2} A_{3} A_{4} B_{1} B_{2} B_{3} B_{4}\right)
$$

which is the desired consequence of (35). Thus, we have proved (24).

Next, we show that (25) must hold. Lemma 9 and $I_{2}$ yield (46), shown at the bottom of the page. Incorporating $I_{1}$ and $\left(e \perp A_{1} \mid A_{2} A_{3} A_{4} B_{1} B_{2} B_{3} B_{4}\right)(45)$, and cancelling some equal terms yields

$$
\begin{aligned}
& \frac{p\left(\mathbf{A}_{4}^{\prime} \mid \mathbf{A}_{1}^{\prime} \mathbf{A}_{2}^{*} \mathbf{A}_{3}^{*}\right) p\left(\mathbf{A}_{1}^{\prime} \mathbf{A}_{2}^{*} \mathbf{A}_{3}^{*}\right) p\left(\mathbf{B}_{1}^{*} \mathbf{B}_{2}^{*} \mathbf{B}_{3}^{*} \mathbf{B}_{4}^{*}\right)}{p\left(\mathbf{A}_{4}^{\prime} \mid \mathbf{A}_{1}^{\prime \prime} \mathbf{A}_{2}^{*} \mathbf{A}_{3}^{*}\right) p\left(\mathbf{A}_{1}^{\prime \prime} \mathbf{A}_{2}^{*} \mathbf{A}_{3}^{*}\right) p\left(\mathbf{B}_{1}^{*} \mathbf{B}_{2}^{*} \mathbf{B}_{3}^{*} \mathbf{B}_{4}^{*}\right)} \\
& \quad=\frac{p\left(\mathbf{A}_{4}^{\prime \prime} \mid \mathbf{A}_{1}^{\prime} \mathbf{A}_{2}^{*} \mathbf{A}_{3}^{*}\right) p\left(\mathbf{A}_{1}^{\prime} \mathbf{A}_{2}^{*} \mathbf{A}_{3}^{*}\right) p\left(\mathbf{B}_{1}^{*} \mathbf{B}_{2}^{*} \mathbf{B}_{3}^{*} \mathbf{B}_{4}^{*}\right)}{p\left(\mathbf{A}_{4}^{\prime \prime} \mid \mathbf{A}_{1}^{\prime \prime} \mathbf{A}_{2}^{*} \mathbf{A}_{3}^{*}\right) p\left(\mathbf{A}_{1}^{\prime \prime} \mathbf{A}_{2}^{*} \mathbf{A}_{3}^{*}\right) p\left(\mathbf{B}_{1}^{*} \mathbf{B}_{2}^{*} \mathbf{B}_{3}^{*} \mathbf{B}_{4}^{*}\right)} .
\end{aligned}
$$

Further cancellation of equal terms yields

$$
\frac{p\left(\mathbf{A}_{4}^{\prime} \mid \mathbf{A}_{1}^{\prime} \mathbf{A}_{2}^{*} \mathbf{A}_{3}^{*}\right)}{p\left(\mathbf{A}_{4}^{\prime \prime} \mid \mathbf{A}_{1}^{\prime} \mathbf{A}_{2}^{*} \mathbf{A}_{3}^{*}\right)}=\frac{p\left(\mathbf{A}_{4}^{\prime} \mid \mathbf{A}_{1}^{\prime \prime} \mathbf{A}_{2}^{*} \mathbf{A}_{3}^{*}\right)}{p\left(\mathbf{A}_{4}^{\prime \prime} \mid \mathbf{A}_{1}^{\prime \prime} \mathbf{A}_{2}^{*} \mathbf{A}_{3}^{*}\right)} .
$$

Thus, $p\left(\mathbf{A}_{4}^{\prime} \mid \mathbf{A}_{1}^{\prime} \mathbf{A}_{2}^{*} \mathbf{A}_{3}^{*}\right)=p\left(\mathbf{A}_{4}^{\prime} \mid \mathbf{A}_{1}^{\prime \prime} \mathbf{A}_{2}^{*} \mathbf{A}_{3}^{*}\right)$ for every instance $\mathbf{A}_{1}^{\prime}, \mathbf{A}_{1}^{\prime \prime}$, and $\mathbf{A}_{4}^{\prime}$. That is, $\left(A_{4} \perp A_{1} \mid \mathbf{A}_{2}^{*} \mathbf{A}_{3}^{*}\right)$ holds. Because $\mathbf{A}_{2}^{*}$ and $\mathbf{A}_{3}^{*}$ are arbitrary instances, $\left(A_{4} \perp A_{1} \mid A_{2} A_{3}\right)$ follows.

Next, we show that (26) must hold. Lemma 9 and $I_{2}$ yield (47), shown at the bottom of the page. Incorporating $I_{1},\left(A_{1} \perp A_{4} \mid A_{2} A_{3}\right)$, and $\left(e \perp A_{1} \mid A_{2} A_{3} A_{4} B_{1} B_{2} B_{3} B_{4}\right)$ and cancelling some equal terms yields (48), shown at the bottom of the page. Further cancellation of equal terms yields

$$
\frac{p\left(\mathbf{A}_{3}^{\prime} \mid \mathbf{A}_{1}^{\prime} \mathbf{A}_{2}^{*}\right)}{p\left(\mathbf{A}_{3}^{\prime \prime} \mid \mathbf{A}_{1}^{\prime} \mathbf{A}_{2}^{*}\right)}=\frac{p\left(\mathbf{A}_{3}^{\prime} \mid \mathbf{A}_{1}^{\prime \prime} \mathbf{A}_{2}^{*}\right)}{p\left(\mathbf{A}_{3}^{\prime \prime} \mid \mathbf{A}_{1}^{\prime \prime} \mathbf{A}_{2}^{*}\right)} .
$$

Thus, $p\left(\mathbf{A}_{3}^{\prime} \mid \mathbf{A}_{1}^{\prime} \mathbf{A}_{2}^{*}\right)=p\left(\mathbf{A}_{3}^{\prime} \mid \mathbf{A}_{1}^{\prime \prime} \mathbf{A}_{2}^{*}\right)$ for every instance $\mathbf{A}_{1}^{\prime}, \mathbf{A}_{1}^{\prime \prime}$, and $\mathbf{A}_{3}^{\prime}$. That is, $\left(A_{3} \perp A_{1} \mid \mathbf{A}_{2}^{*}\right)$ holds. Because $\mathbf{A}_{2}^{*}$ is an arbitrary instance, $\left(A_{3} \perp A_{1} \mid A_{2}\right)$ follows.

Finally, we must show that (27) holds. Lemma 9 and $I_{3}$ yield (49), shown at the bottom of the page. Incorporating ( $\left.e \perp A_{1} \mid A_{2} A_{3} A_{4} B_{1} B_{2} B_{3} B_{4}\right), I_{1}, I_{3},\left(A_{1} \perp A_{4} \mid A_{2} A_{3}\right)$, and $\left(A_{1} \perp A_{3} \mid A_{2}\right)$ and cancelling some equal terms yields

$$
\begin{aligned}
& \underline{p\left(\mathbf{e} \mid \mathbf{A}_{1}^{\prime} \mathbf{A}_{2}^{*} \mathbf{A}_{3}^{*} \mathbf{A}_{4}^{\prime} \mathbf{B}_{1}^{*} \mathbf{B}_{2}^{*} \mathbf{B}_{3}^{*} \mathbf{B}_{4}^{*}\right) p\left(\mathbf{A}_{1}^{\prime} \mathbf{A}_{2}^{*} \mathbf{A}_{3}^{*} \mathbf{A}_{4}^{\prime} \mathbf{B}_{1}^{*} \mathbf{B}_{2}^{*} \mathbf{B}_{3}^{*} \mathbf{B}_{4}^{*}\right)} \\
& p\left(\mathbf{e} \mid \mathbf{A}_{1}^{\prime \prime} \mathbf{A}_{2}^{*} \mathbf{A}_{3}^{*} \mathbf{A}_{4}^{\prime} \mathbf{B}_{1}^{*} \mathbf{B}_{2}^{*} \mathbf{B}_{3}^{*} \mathbf{B}_{4}^{*}\right) p\left(\mathbf{A}_{1}^{\prime \prime} \mathbf{A}_{2}^{*} \mathbf{A}_{3}^{*} \mathbf{A}_{4}^{\prime} \mathbf{B}_{1}^{*} \mathbf{B}_{2}^{*} \mathbf{B}_{3}^{*} \mathbf{B}_{4}^{*}\right) \\
& =\frac{p\left(\mathbf{e} \mid \mathbf{A}_{1}^{\prime} \mathbf{A}_{2}^{*} \mathbf{A}_{3}^{*} \mathbf{A}_{4}^{\prime \prime} \mathbf{B}_{1}^{*} \mathbf{B}_{2}^{*} \mathbf{B}_{3}^{*} \mathbf{B}_{4}^{*}\right) p\left(\mathbf{A}_{1}^{\prime} \mathbf{A}_{2}^{*} \mathbf{A}_{3}^{*} \mathbf{A}_{4}^{\prime \prime} \mathbf{B}_{1}^{*} \mathbf{B}_{2}^{*} \mathbf{B}_{3}^{*} \mathbf{B}_{4}^{*}\right)}{p\left(\mathbf{e} \mid \mathbf{A}_{1}^{\prime \prime} \mathbf{A}_{2}^{*} \mathbf{A}_{3}^{*} \mathbf{A}_{4}^{\prime \prime} \mathbf{B}_{1}^{*} \mathbf{B}_{2}^{*} \mathbf{B}_{3}^{*} \mathbf{B}_{4}^{*}\right) p\left(\mathbf{A}_{1}^{\prime \prime} \mathbf{A}_{2}^{*} \mathbf{A}_{3}^{*} \mathbf{A}_{4}^{\prime \prime} \mathbf{B}_{1}^{*} \mathbf{B}_{2}^{*} \mathbf{B}_{3}^{*} \mathbf{B}_{4}^{*}\right)} \\
& p\left(\mathbf{e} \mid \mathbf{A}_{1}^{\prime} \mathbf{A}_{2}^{*} \mathbf{A}_{3}^{\prime} \mathbf{A}_{4}^{*} \mathbf{B}_{1}^{*} \mathbf{B}_{2}^{*} \mathbf{B}_{3}^{*} \mathbf{B}_{4}^{*}\right) p\left(\mathbf{A}_{1}^{\prime} \mathbf{A}_{2}^{*} \mathbf{A}_{3}^{\prime} \mathbf{A}_{4}^{*} \mathbf{B}_{1}^{*} \mathbf{B}_{2}^{*} \mathbf{B}_{3}^{*} \mathbf{B}_{4}^{*}\right) \\
& p\left(\mathbf{e} \mid \mathbf{A}_{1}^{\prime \prime} \mathbf{A}_{2}^{*} \mathbf{A}_{3}^{\prime} \mathbf{A}_{4}^{*} \mathbf{B}_{1}^{*} \mathbf{B}_{2}^{*} \mathbf{B}_{3}^{*} \mathbf{B}_{4}^{*}\right) p\left(\mathbf{A}_{1}^{\prime \prime} \mathbf{A}_{2}^{*} \mathbf{A}_{3}^{\prime} \mathbf{A}_{4}^{*} \mathbf{B}_{1}^{*} \mathbf{B}_{2}^{*} \mathbf{B}_{3}^{*} \mathbf{B}_{4}^{*}\right) \\
& =\frac{p\left(\mathbf{e} \mid \mathbf{A}_{1}^{\prime} \mathbf{A}_{2}^{*} \mathbf{A}_{3}^{\prime \prime} \mathbf{A}_{4}^{*} \mathbf{B}_{1}^{*} \mathbf{B}_{2}^{*} \mathbf{B}_{3}^{*} \mathbf{B}_{4}^{*}\right) p\left(\mathbf{A}_{1}^{\prime} \mathbf{A}_{2}^{*} \mathbf{A}_{3}^{\prime \prime} \mathbf{A}_{4}^{*} \mathbf{B}_{1}^{*} \mathbf{B}_{2}^{*} \mathbf{B}_{3}^{*} \mathbf{B}_{4}^{*}\right)}{p\left(\mathbf{e} \mid \mathbf{A}_{1}^{\prime \prime} \mathbf{A}_{2}^{*} \mathbf{A}_{3}^{\prime \prime} \mathbf{A}_{4}^{*} \mathbf{B}_{1}^{*} \mathbf{B}_{2}^{*} \mathbf{B}_{3}^{*} \mathbf{B}_{4}^{*}\right) p\left(\mathbf{A}_{1}^{\prime \prime} \mathbf{A}_{2}^{*} \mathbf{A}_{3}^{\prime \prime} \mathbf{A}_{4}^{*} \mathbf{B}_{1}^{*} \mathbf{B}_{2}^{*} \mathbf{B}_{3}^{*} \mathbf{B}_{4}^{*}\right)} \\
& \frac{p\left(\mathbf{A}_{4}^{*} \mid \mathbf{A}_{2}^{*} \mathbf{A}_{3}^{\prime}\right) p\left(\mathbf{A}_{3}^{\prime} \mid \mathbf{A}_{1}^{\prime} \mathbf{A}_{2}^{*}\right) p\left(\mathbf{A}_{1}^{\prime} \mathbf{A}_{2}^{*}\right) p\left(\mathbf{B}_{1}^{*} \mathbf{B}_{2}^{*} \mathbf{B}_{3}^{*} \mathbf{B}_{4}^{*}\right)}{p\left(\mathbf{A}_{4}^{*} \mid \mathbf{A}_{2}^{*} \mathbf{A}_{3}^{\prime}\right) p\left(\mathbf{A}_{3}^{\prime} \mid \mathbf{A}_{1}^{\prime \prime} \mathbf{A}_{2}^{*}\right) p\left(\mathbf{A}_{1}^{\prime \prime} \mathbf{A}_{2}^{*}\right) p\left(\mathbf{B}_{1}^{*} \mathbf{B}_{2}^{*} \mathbf{B}_{3}^{*} \mathbf{B}_{4}^{*}\right)} \\
& =\frac{p\left(\mathbf{A}_{4}^{*} \mid \mathbf{A}_{2}^{*} \mathbf{A}_{3}^{\prime \prime}\right) p\left(\mathbf{A}_{3}^{\prime \prime} \mid \mathbf{A}_{1}^{\prime} \mathbf{A}_{2}^{*}\right) p\left(\mathbf{A}_{1}^{\prime} \mathbf{A}_{2}^{*}\right) p\left(\mathbf{B}_{1}^{*} \mathbf{B}_{2}^{*} \mathbf{B}_{3}^{*} \mathbf{B}_{4}^{*}\right)}{p\left(\mathbf{A}_{4}^{*} \mid \mathbf{A}_{2}^{*} \mathbf{A}_{3}^{\prime \prime}\right) p\left(\mathbf{A}_{3}^{\prime \prime} \mid \mathbf{A}_{1}^{\prime \prime} \mathbf{A}_{2}^{*}\right) p\left(\mathbf{A}_{1}^{\prime \prime} \mathbf{A}_{2}^{*}\right) p\left(\mathbf{B}_{1}^{*} \mathbf{B}_{2}^{*} \mathbf{B}_{3}^{*} \mathbf{B}_{4}^{*}\right)} \\
& \frac{p\left(\overline{\mathbf{e}} \mid \mathbf{A}_{1}^{\prime} \mathbf{A}_{2}^{\prime} \mathbf{A}_{3}^{*} \mathbf{A}_{4}^{*} \mathbf{B}_{1}^{*} \mathbf{B}_{2}^{*} \mathbf{B}_{3}^{*} \mathbf{B}_{4}^{*}\right) p\left(\mathbf{A}_{1}^{\prime} \mathbf{A}_{2}^{\prime} \mathbf{A}_{3}^{*} \mathbf{A}_{4}^{*} \mathbf{B}_{1}^{*} \mathbf{B}_{2}^{*} \mathbf{B}_{3}^{*} \mathbf{B}_{4}^{*}\right)}{p\left(\overline{\mathbf{e}} \mid \mathbf{A}^{\prime \prime} \mathbf{A}_{2}^{\prime} \mathbf{A}_{3}^{*} \mathbf{A}^{*} \mathbf{B}^{*} \mathbf{B}_{2}^{*} \mathbf{B}^{*} \mathbf{B}^{*}\right) p\left(\mathbf{A}_{1}^{\prime \prime} \mathbf{A}_{2}^{\prime} \mathbf{A}^{*} \mathbf{A}^{*} \mathbf{B}^{*} \mathbf{B}_{2}^{*} \mathbf{B}^{*} \mathbf{B}^{*}\right)} \\
& =\frac{p\left(\overline{\mathbf{e}} \mid \mathbf{A}_{1}^{\prime} \mathbf{A}_{2}^{\prime \prime} \mathbf{A}_{3}^{*} \mathbf{A}_{4}^{*} \mathbf{B}_{1}^{*} \mathbf{B}_{2}^{*} \mathbf{B}_{3}^{*} \mathbf{B}_{4}^{*}\right) p\left(\mathbf{A}_{1}^{\prime} \mathbf{A}_{2}^{\prime \prime} \mathbf{A}_{3}^{*} \mathbf{A}_{4}^{*} \mathbf{B}_{1}^{*} \mathbf{B}_{2}^{*} \mathbf{B}_{3}^{*} \mathbf{B}_{4}^{*}\right)}{p\left(\overline{\mathbf{e}} \mid \mathbf{A}_{1}^{\prime \prime} \mathbf{A}_{2}^{\prime \prime} \mathbf{A}_{3}^{*} \mathbf{A}_{4}^{*} \mathbf{B}_{1}^{*} \mathbf{B}_{2}^{*} \mathbf{B}_{3}^{*} \mathbf{B}_{4}^{*}\right) p\left(\mathbf{A}_{1}^{\prime \prime} \mathbf{A}_{2}^{\prime \prime} \mathbf{A}_{3}^{*} \mathbf{A}_{4}^{*} \mathbf{B}_{1}^{*} \mathbf{B}_{2}^{*} \mathbf{B}_{3}^{*} \mathbf{B}_{4}^{*}\right)} \\
& \frac{p\left(\mathbf{A}_{4}^{*} \mid \mathbf{A}_{2}^{\prime} * \mathbf{A}_{3}^{*}\right) p\left(\mathbf{A}_{3}^{*} \mid \mathbf{A}_{1}^{\prime} \mathbf{A}_{2}^{\prime}\right) p\left(\mathbf{A}_{2}^{\prime} \mid \mathbf{A}_{1}^{\prime}\right) p\left(\mathbf{A}_{1}^{\prime}\right) p\left(\mathbf{B}_{1}^{*} \mathbf{B}_{2}^{*} \mathbf{B}_{3}^{*} \mathbf{B}_{4}^{*}\right)}{p\left(\mathbf{A}_{4}^{*} \mid \mathbf{A}_{2}^{\prime} * \mathbf{A}_{3}^{*}\right) p\left(\mathbf{A}_{3}^{*} \mid \mathbf{A}_{1}^{\prime \prime} \mathbf{A}_{2}^{\prime}\right) p\left(\mathbf{A}_{2}^{\prime} \mid \mathbf{A}_{1}^{\prime \prime}\right) p\left(\mathbf{A}_{1}^{\prime \prime}\right) p\left(\mathbf{B}_{1}^{*} \mathbf{B}_{2}^{*} \mathbf{B}_{3}^{*} \mathbf{B}_{4}^{*}\right)} \\
& =\frac{p\left(\mathbf{A}_{4}^{*} \mid \mathbf{A}_{2}^{\prime \prime} * \mathbf{A}_{3}^{*}\right) p\left(\mathbf{A}_{3}^{*} \mid \mathbf{A}_{1}^{\prime} \mathbf{A}_{2}^{\prime \prime}\right) p\left(\mathbf{A}_{2}^{\prime \prime} \mid \mathbf{A}_{1}^{\prime}\right) p\left(\mathbf{A}_{1}^{\prime}\right) p\left(\mathbf{B}_{1}^{*} \mathbf{B}_{2}^{*} \mathbf{B}_{3}^{*} \mathbf{B}_{4}^{*}\right)}{p\left(\mathbf{A}_{4}^{*} \mid \mathbf{A}_{2}^{\prime \prime} * \mathbf{A}_{3}^{*}\right) p\left(\mathbf{A}_{3}^{*} \mid \mathbf{A}_{1}^{\prime \prime} \mathbf{A}_{2}^{\prime \prime}\right) p\left(\mathbf{A}_{2}^{\prime \prime} \mid \mathbf{A}_{1}^{\prime \prime}\right) p\left(\mathbf{A}_{1}^{\prime \prime}\right) p\left(\mathbf{B}_{1}^{*} \mathbf{B}_{2}^{*} \mathbf{B}_{3}^{*} \mathbf{B}_{4}^{*}\right)}
\end{aligned}
$$


(50), shown at the bottom of the preceding page. Further cancellation of equal terms yields

$$
\frac{p\left(\mathbf{A}_{2}^{\prime} \mid \mathbf{A}_{1}^{\prime}\right)}{p\left(\mathbf{A}_{2}^{\prime \prime} \mid \mathbf{A}_{1}^{\prime}\right)}=\frac{p\left(\mathbf{A}_{2}^{\prime} \mid \mathbf{A}_{1}^{\prime \prime}\right)}{p\left(\mathbf{A}_{2}^{\prime \prime} \mid \mathbf{A}_{1}^{\prime \prime}\right)}
$$

Thus, $p\left(\mathbf{A}_{2}^{\prime} \mid \mathbf{A}_{1}^{\prime}\right)=p\left(\mathbf{A}_{2}^{\prime} \mid \mathbf{A}_{1}^{\prime \prime}\right)$ for every instance $\mathbf{A}_{1}^{\prime}, \mathbf{A}_{1}^{\prime \prime}$, and $\mathbf{A}_{2}^{\prime}$. That is, $\left(A_{2} \perp A_{1} \mid \emptyset\right)$ holds.

\section{REFERENCES}

[1] A. P. Dawid, "Conditional independence in statistical theory," J. Roy. Stat. Soc. B, vol. 41, pp. 1-31, 1979.

[2] D. Geiger, "Graphoids: A qualitative framework for probabilistic inference," Ph.D. dissertation, Dept. Comput. Sci., Univ. Calif., Los Angeles, 1990.

[3] D. Geiger and D. Heckerman, "Knowledge representation and inference in similarity networks and Bayesian multinets," Artif. Intell., vol. 82, pp. $45-74,1996$.

[4] _ "Separable and transitive graphoids," in Proc. 6th Conf. Uncertainty Artificial Intelligence $D, 1990$.

[5] D. Geiger, T. S. Verma, and J. Pearl, "Identifying independence in Bayesian networks," Networks, vol. 20, pp. 507-534, 1990.

[6] D. Geiger and J. Pearl, "On the logic of causal models," in Uncertainty in Artificial Intelligence 4, R. D. Shachter, T. S. Levitt, L. N. Kanal, and J. F. Lemmer, Eds. Amsterdam, The Netherlands, Elsevier, 1990, pp. 3-12.

[7] D. Heckerman, Probabilistic Similarity Networks. Cambridge, MA: MIT Press, 1991.

[8] S. L. Lauritzen, A. P. Dawid, B. N. Larsen, and H. G. Leimer, "Independence properties of directed Markov fields," Networks, vol. 20, pp. 491-550, 1990.

[9] J. Pearl, Probabilistic Reasoning in Intelligent Systems: Networks of Plausible Inference. San Mateo, CA: Morgan Kaufmann, 1988.

[10] J. Pearl and A. Paz, "Graphoids: A graph-based logic for reasoning about relevance relations," in Advances in Artificial Intelligence-II, B. Du Boulay et al., Eds. Amsterdam, The Netherlands: North-Holland, 1990, pp. $357-363$.
[11] R. Shachter, "An ordered examination of influence diagrams," Networks, vol. 20 , pp. $535-564,1990$.

[12] W. Spohn, "Stochastic independence, causal independence, and shieldability," J. Phil. Logic, vol. 9, pp. 73-99, 1980

[13] T. Verma and J. Pearl, "Causal networks: Semantics and expressiveness," in Proc. 4th Workshop Uncertainty Artificial Intelligence, Minneapolis, MN, 1988, pp. 352-359; also, T. Verma, Tech. Rep. R-65, Cognitive Systems Lab., Univ. Calif., Los Angeles, 1986.

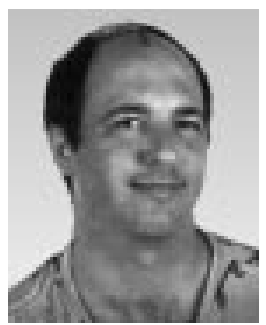

Dan Geiger was born in Haifa, Israel, in 1959. $\mathrm{He}$ received the B.Sc. degree in computer engineering and the B.A. degree in mathematics from the Technion-Israel Institute of Technology, Haifa, in 1985, and the M.Sc. and Ph.D. degrees from the University of California, Los Angeles, in 1987 and 1990, respectively.

His research is focused on the study of conditional independence, learning Bayesian networks, discrete optimization problems in probability and statistics, and genetic linkage analysis.

Dr. Geiger received the Technion's 1997 Taub Award for excellence in research and the 1997 AAAI Best Paper Award for "A practical algorithm for finding optimal triangulations."

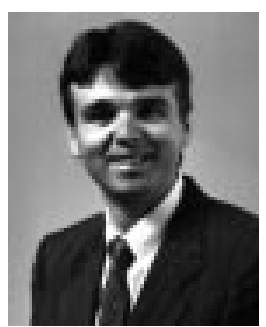

David Heckerman received the Ph.D. degree in medical information sciences from Stanford University, Stanford, CA, in 1990.

Since 1992, he has been a Senior Researcher at Microsoft Research, Redmond, WA, where he has been developing methods for learning from data using graphical models.

Dr. Heckerman received the outstanding computer-science dissertation award from the ACM in 1990 for his paper "Probabilistic similarity networks." 